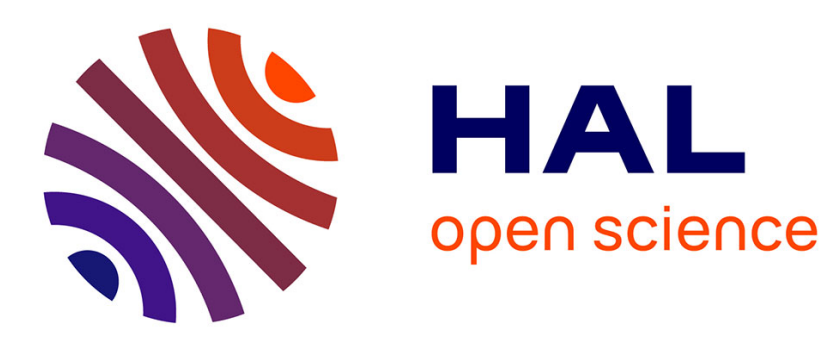

\title{
A discontinuous skeletal method for the viscosity-dependent Stokes problem
}

Daniele Di Pietro, Alexandre Ern, Alexander Linke, Friedhelm Schieweck

\section{To cite this version:}

Daniele Di Pietro, Alexandre Ern, Alexander Linke, Friedhelm Schieweck. A discontinuous skeletal method for the viscosity-dependent Stokes problem. Computer Methods in Applied Mechanics and Engineering, 2016, 306, pp.175-195. 10.1016/j.cma.2016.03.033 . hal-01244387v2

\section{HAL Id: hal-01244387 \\ https://hal.science/hal-01244387v2}

Submitted on 2 May 2016

HAL is a multi-disciplinary open access archive for the deposit and dissemination of scientific research documents, whether they are published or not. The documents may come from teaching and research institutions in France or abroad, or from public or private research centers.
L'archive ouverte pluridisciplinaire HAL, est destinée au dépôt et à la diffusion de documents scientifiques de niveau recherche, publiés ou non, émanant des établissements d'enseignement et de recherche français ou étrangers, des laboratoires publics ou privés. 


\title{
A discontinuous skeletal method for the viscosity-dependent Stokes problem
}

\author{
Daniele A. Di Pietro*1, Alexandre Ern ${ }^{\dagger 2}$, Alexander Linke ${ }^{\ddagger 3}$, and Friedhelm Schieweck ${ }^{\S 4}$ \\ ${ }^{1}$ University Montpellier, Institut Montpelliérain Alexander Grothendieck, 34095 Montpellier Cedex 5, France \\ ${ }^{2}$ Université Paris-Est, CERMICS (ENPC), 77455 Marne la Vallee Cedex 2, France \\ ${ }^{3}$ Weierstrass Institute for Applied Analysis and Stochastics, 10117 Berlin, Germany \\ ${ }^{4}$ Institut für Analysis und Numerik, Otto-von-Guericke-Universität Magdeburg, Postfach 4120, D-39016 Magdeburg, \\ Germany
}

May 2, 2016

\begin{abstract}
We devise and analyze arbitrary-order nonconforming methods for the discretization of the viscosity-dependent Stokes equations on simplicial meshes. We keep track explicitly of the viscosity and aim at pressure-robust schemes that can deal with the practically relevant case of body forces with large curl-free part in a way that the discrete velocity error is not spoiled by large pressures. The method is inspired from the recent Hybrid High-Order (HHO) methods for linear elasticity. After elimination of the auxiliary variables by static condensation, the linear system to be solved involves only discrete face-based velocities, which are polynomials of degree $k \geqslant 0$, and cell-wise constant pressures. Our main result is a pressure-independent energy-error estimate on the velocity of order $(k+1)$. The main ingredient to achieve pressure-independence is the use of a divergencepreserving velocity reconstruction operator in the discretization of the body forces. We also prove an $L^{2}$-pressure estimate of order $(k+1)$ and an $L^{2}$-velocity estimate of order $(k+2)$, the latter under elliptic regularity. The local mass and momentum conservation properties of the discretization are also established. Finally, two- and three-dimensional numerical results are presented to support the analysis.
\end{abstract}

Keywords: Stokes problem, mixed methods, curl-free forces, higher-order reconstruction, superconvergence, hybrid discontinuous Galerkin method, static condensation

2000 Mathematics Subject Classification (MSC): 65N12, 65N30, 76D07

\section{Introduction}

The arbitrary-order nonconforming methods analyzed in this work are inspired from the Hybrid HighOrder (HHO) methods recently introduced in [16] for quasi-incompressible linear elasticity and in [17] for diffusion problems. In a nutshell, HHO methods are formulated using cell- and face-based discrete unknowns that are typically polynomials of some order $k \geqslant 0$. These methods rely upon two key ingredients: a high-order reconstruction operator inside cells from the cell- and face-based unknowns, and a stabilization operator linking locally cell- and face-based unknowns while preserving the high order of the reconstruction. The discrete problem is assembled cell-wise, and cell-based unknowns can be eliminated by static condensation, leading for diffusion problems to a symmetric, positive definite linear system coupling the face-based unknowns. To emphasize the fact that the only globally coupled variables are broken polynomials on the mesh skeleton, we have recently introduced [13] the nomenclature discontinuous skeletal methods. HHO methods support general meshes and lead to energy error estimates of order $(k+1)$ for smooth solutions and to super-closeness of order $(k+2)$ for the primal variable. These

\footnotetext{
*daniele.di-pietro@umontpellier.fr

$\dagger$ ern@cermics.enpc.fr

$\ddagger$ alexander.linke@wias-berlin.de, ORCID: 0000-0002-0165-2698

§schiewec@ovgu.de
} 
methods are also locally conservative, see [15], and, as detailed in [9], close connections exist with the hybridizable discontinuous Galerkin (HDG) methods, as well as with the High-Order Mimetic methods from [33]. The most salient differences are the choice of stabilization and that of the spaces to reconstruct the dual variable (the flux) in HHO methods with respect to HDG.

The aim of this paper is to devise and analyze a discontinuous skeletal method to discretize the viscositydependent Stokes equations on three-dimensional simplicial meshes. We keep track explicitly of the dependence on the (kinematic) viscosity in the analysis so as to address the practically important issue of body forces with a large curl-free part, as encountered in more complex flows owing to the nonlinear convection term [28], exterior forces like buoyancy [20,25], or Coriolis forces [32, 25] for examples. Our discretization of the Stokes equations hinges on face-based discrete velocities, which are vector-valued polynomials of degree $k \geqslant 0$, and cell-based discrete constant pressures. All the remaining unknowns that are considered in the derivation of the method, namely cell-based discrete velocities and higher-order discrete pressures, can be eliminated by static condensation. Our main result, see Theorem 4 below, is an energy-norm velocity error estimate of order $(k+1)$ which is independent of the exact pressure (and, thus, of the viscosity). Furthermore, $L^{2}$-error estimates of order $(k+1)$ and $(k+2)$ are obtained for the pressure and the velocity, respectively, the latter under the usual elliptic regularity assumption. We also identify the local mass and momentum conservation properties of the method. Some high-order methods using $H^{1}$-conforming velocities and pressures in 2D/3D and addressing robustness against large curl-free forces appeared recently in [31] (together with some nonconforming methods in 2D). The present method adopts instead a nonconforming approach for the velocity (in 2D/3D). Possible advantages include local momentum and mass balance and more compact stencils; the locality of the present method could also be exploited in an $h p$-adaptive context.

While the discretization of the viscous term and the incompressibility constraint follow along the lines of $[17,16,1]$, the key novelty introduced in the present work is the construction of a high-order, divergence-preserving velocity reconstruction operator on simplicial meshes, whose role is to ensure an exact balance of the curl-free part of all the forces in the momentum equation $[30,25]$ at the discrete level. In other words, this operator allows us to reestablish the $L^{2}$-orthogonality in the momentum balance between discretely divergence-free velocity fields and curl-free vector fields. This property is, in turn, the key ingredient to achieve viscosity-independent velocity error estimates. It is well-known that nearly all classical discretization methods for the incompressible Stokes equations fail to achieve such a property. This difficuly is traditionally tempered, but not cured, by adding grad-div stabilization $[37,36,22,5]$. An alternative approach avoiding this stabilization - avoiding the need to tune stabilization parameters (!) - is the variational crime introduced recently in $[29,30]$ in the lowest-order case with Crouzeix-Raviart $/ \mathbb{P}_{0}$ mixed finite elements. The present work can be viewed as a higher-order generalization of $[29,30]$. We also mention [4] for a robust treatment of body forces in the context of (lowest-order) Compatible Discrete Operator (CDO) schemes.

Several high-order discretizations of the Stokes equations using face-based velocities as discrete unknowns have been developed recently. In [10], Cockburn and Gopalakrishnan derive HDG methods for a vorticity-velocity-pressure formulation of the Stokes equations and describe several hybridization procedures corresponding to different choices of the globally coupled unknowns. In [35], Nguyen et al. develop an HDG method for a velocity-pressure-gradient formulation of the Stokes equations for which a velocity reconstruction that converges with order $(k+2)$ in the $L^{2}$-norm can be obtained by a cell-wise post-processing when polynomials of degree $k \geqslant 1$ are used. Similar convergence results are obtained for the HDG method developed by Wang and Khoo [41] for interface problems with discontinuous viscosity and variable surface tension. In [26], Labeur and Wells present an HDG method where the velocity unknowns are polynomials of degree $k$ at elements and faces. We also refer to Cockburn and Shi [11] for an overview of HDG methods for Stokes flows. Other methods using face-based polynomial velocities as main unknowns include: the method of Egger and Waluga [18], where polynomials of degree $k$ and $(k-1)$ are used for the velocity and the pressure, respectively, and a $h p$-convergence analysis is carried out; the hybridized finite element method of Jeon et al. [23, 24] based on local Dirichlet solves; the method of $\mathrm{Mu}$ et al. [34]. The present work is the first one that addresses the robust treatment of large curl-free forces using face-based discrete velocities of arbitrary polynomial arbitrary-order; we also mention the recent work [27] in the HDG context.

This paper is organized as follows. In Section 2, we present the model problem and introduce some mesh- 
related definitions. The discretization is devised in Section 3, leading to the discrete problem (24) below. Our main results, including stability, error estimates, and local conservation properties, are stated in Section 4. Two- and three-dimensional numerical results, are treated in Section 5 whereas computational aspects, including static condensation, are discussed in Section 6. Finally, the proofs of our main results are collected in Section 7 .

\section{Preliminaries}

\subsection{Model problem}

Let $\Omega \subset \mathbb{R}^{d}, d \geqslant 2$, denote an open bounded and connected domain with a polygonal boundary $\Gamma$ and $\boldsymbol{f} \in \boldsymbol{L}^{2}(\Omega):=L^{2}(\Omega)^{d}$ be a given vector field. We consider the model problem: Find the velocity $\boldsymbol{u}: \Omega \rightarrow \mathbb{R}^{d}$ and the pressure $p: \Omega \rightarrow \mathbb{R}$ such that

$$
\begin{aligned}
-\nu \triangle \boldsymbol{u}+\nabla p & =\boldsymbol{f} & & \text { in } \Omega, \\
\operatorname{div} \boldsymbol{u} & =0 & & \text { in } \Omega, \\
\boldsymbol{u} & =\mathbf{0} & & \text { on } \Gamma,
\end{aligned}
$$

where $\nu>0$ is a constant viscosity parameter. For any subset $X \subset \bar{\Omega}$, we respectively denote by $(\cdot, \cdot)_{X}$ and $\|\cdot\|_{X}$ the standard inner product and norm in $L^{2}(X)$, and we omit the subscript whenever $X=\Omega$. The same notation is used in the vector- and tensor-valued cases. Letting $\boldsymbol{H}_{0}^{1}(\Omega):=H_{0}^{1}(\Omega)^{d}$ and $L_{0}^{2}(\Omega):=\left\{q \in L^{2}(\Omega) \mid \int_{\Omega} q=0\right\}$, the weak formulation of problem (1) reads: Find $(\boldsymbol{u}, p) \in \boldsymbol{H}_{0}^{1}(\Omega) \times L_{0}^{2}(\Omega)$ such that

$$
\begin{aligned}
\nu a(\boldsymbol{u}, \boldsymbol{v})+b(\boldsymbol{v}, p) & =(\boldsymbol{f}, \boldsymbol{v}) & & \forall \boldsymbol{v} \in \boldsymbol{H}_{0}^{1}(\Omega), \\
b(\boldsymbol{u}, q) & =0 & & \forall q \in L_{0}^{2}(\Omega),
\end{aligned}
$$

with bilinear forms $a$ and $b$ defined by

$$
a(\boldsymbol{u}, \boldsymbol{v}):=(\nabla \boldsymbol{u}, \nabla \boldsymbol{v}), \quad b(\boldsymbol{v}, p):=-(\operatorname{div} \boldsymbol{v}, p) .
$$

The well-posedness of problem (2) classically hinges on the coercivity of the bilinear form $a$ together with the inf-sup stability of the bilinear form $b$ (cf., e.g., [21, Section 2.2], [19, Theorem 4.3]), or [3, Section 8.2].

\subsection{Meshes}

Denote by $\mathcal{H} \subset \mathbb{R}_{*}^{+}$a countable set of meshsizes having 0 as its unique accumulation point. We consider an $h$-refined mesh sequence $\left(\mathcal{T}_{h}\right)_{h \in \mathcal{H}}$ where, for all $h \in \mathcal{H}, \mathcal{T}_{h}=\{T\}$ is a matching simplicial mesh characterized by the scalar $h:=\max _{T \in \mathcal{T}_{h}} h_{T}$ with $h_{T}$ denoting the diameter of the element $T$. The mesh sequence is assumed to be shape-regular in the sense of Ciarlet [7]. Interfaces are collected in the set $\mathcal{F}_{h}^{\mathrm{i}}$, boundary faces in $\mathcal{F}_{h}^{\mathrm{b}}$, and we let $\mathcal{F}_{h}:=\mathcal{F}_{h}^{\mathrm{i}} \cup \mathcal{F}_{h}^{\mathrm{b}}$. The diameter of a face $F \in \mathcal{F}_{h}$ is denoted by $h_{F}$. For all $T \in \mathcal{T}_{h}, \mathcal{F}_{T}$ denotes the set of faces of $T$ and, for all $F \in \mathcal{F}_{T}, \boldsymbol{n}_{T F}$ is the unit normal to $F$ pointing out of $T$. In what follows, we use the abbreviation $a \lesssim b$ for the inequality $a \leqslant C b$ with generic positive constant $C$ independent of $h$ and of $\nu$.

\section{Key ingredients of the discretization}

All the material contained in this section is devised at the local level; thus, we fix an arbitrary mesh cell $T \in \mathcal{T}_{h}$. 


\subsection{Local velocity space and local reduction map}

Let a polynomial degree $k \geqslant 0$ be fixed. We define the local discrete space for the velocity as

$$
\underline{U}_{T}^{k}:=\mathbb{P}_{k}(T)^{d} \times\left\{\underset{F \in \mathcal{F}_{T}}{\underset{X}{X}} \mathbb{P}_{k}(F)^{d}\right\},
$$

where $\mathbb{P}_{k}(T)$ and $\mathbb{P}_{k}(F)$ denote, respectively, the spaces spanned by the restrictions to $T$ and $F$ of $d$ - and $(d-1)$-variate polynomial functions of total degree $\leqslant k$. We underline symbols to indicate a collection of velocities on elements and faces. Thus, an element $\underline{\boldsymbol{v}}_{T} \in \underline{\boldsymbol{U}}_{T}^{k}$ is called a local collection of velocities on $T$ and is specified as $\underline{\boldsymbol{v}}_{T}:=\left(\boldsymbol{v}_{T},\left(\boldsymbol{v}_{F}\right)_{F \in \mathcal{F}_{T}}\right)$.

We define the local velocity reduction $\operatorname{map} \underline{\boldsymbol{I}}_{T}^{k}: \boldsymbol{H}^{1}(T) \rightarrow \underline{\boldsymbol{U}}_{T}^{k}$ that maps a given vector-valued function $\boldsymbol{v} \in \boldsymbol{H}^{1}(T)$ to a local collection of velocities determined as

$$
\underline{\boldsymbol{I}}_{T}^{k} \boldsymbol{v}:=\left(\boldsymbol{\pi}_{T}^{k} \boldsymbol{v},\left(\boldsymbol{\pi}_{F}^{k} \boldsymbol{v}\right)_{F \in \mathcal{F}_{T}}\right),
$$

where $\boldsymbol{\pi}_{T}^{k}$ and $\boldsymbol{\pi}_{F}^{k}$ denote the $L^{2}$-orthogonal projectors on $\mathbb{P}_{k}(T)^{d}$ and $\mathbb{P}_{k}(F)^{d}$, respectively.

\subsection{Viscous term}

The discretization of the viscous term follows the ideas of [17]. We define the local velocity reconstruction operator $\boldsymbol{r}_{T}^{k+1}: \underline{\boldsymbol{U}}_{T}^{k} \rightarrow \mathbb{P}_{k+1}(T)^{d}$ that maps a local collection of velocities $\underline{\boldsymbol{v}}_{T} \in \underline{\boldsymbol{U}}_{T}^{k}$ to the vector-valued polynomial $\boldsymbol{r}_{T}^{k+1}\left(\underline{\boldsymbol{v}}_{T}\right) \in \mathbb{P}_{k+1}(T)^{d}$ solution of the following Neumann problem: For all $\boldsymbol{w} \in \mathbb{P}_{k+1}(T)^{d}$,

$$
\left(\boldsymbol{\nabla} \boldsymbol{r}_{T}^{k+1}\left(\underline{\boldsymbol{v}}_{T}\right), \boldsymbol{\nabla} \boldsymbol{w}\right)_{T}=\left(\boldsymbol{\nabla} \boldsymbol{v}_{T}, \boldsymbol{\nabla} \boldsymbol{w}\right)_{T}+\sum_{F \in \mathcal{F}_{T}}\left(\boldsymbol{v}_{F}-\boldsymbol{v}_{T}, \boldsymbol{\nabla} \boldsymbol{w} \cdot \boldsymbol{n}_{T F}\right)_{F},
$$

and the mean-value of $\boldsymbol{r}_{T}^{k+1}\left(\underline{\boldsymbol{v}}_{T}\right)$ in $T$ is set equal to that of $\boldsymbol{v}_{T}$. Note that $\boldsymbol{r}_{T}^{k+1}\left(\underline{\boldsymbol{v}}_{T}\right)$ is a vector-valued polynomial field in $T$ one degree higher than the polynomials used in $\underline{U}_{T}^{k}$, and is designed so that the following optimal approximation property holds: For all $\boldsymbol{v} \in \boldsymbol{H}^{k+2}(T)$,

$$
\left\|\boldsymbol{v}-\boldsymbol{r}_{T}^{k+1}\left(\underline{\boldsymbol{I}}_{T}^{k} \boldsymbol{v}\right)\right\|_{T}+h_{T}\left\|\nabla\left(\boldsymbol{v}-\boldsymbol{r}_{T}^{k+1}\left(\underline{\boldsymbol{I}}_{T}^{k} \boldsymbol{v}\right)\right)\right\|_{T} \lesssim h_{T}^{k+2}\|\boldsymbol{v}\|_{\boldsymbol{H}^{k+2}(T)}
$$

The discretization of the viscous term hinges on the local bilinear form $a_{T}$ on $\underline{U}_{T}^{k} \times \underline{\boldsymbol{U}}_{T}^{k}$ such that

$$
a_{T}\left(\underline{\boldsymbol{v}}_{T}, \underline{\boldsymbol{w}}_{T}\right):=\left(\boldsymbol{\nabla} \boldsymbol{r}_{T}^{k+1}\left(\underline{\boldsymbol{v}}_{T}\right), \boldsymbol{\nabla} \boldsymbol{r}_{T}^{k+1}\left(\underline{\boldsymbol{w}}_{T}\right)\right)_{T}+s_{T}\left(\underline{\boldsymbol{v}}_{T}, \underline{\boldsymbol{w}}_{T}\right)
$$

with stabilization bilinear form

$$
s_{T}\left(\underline{\boldsymbol{v}}_{T}, \underline{\boldsymbol{w}}_{T}\right):=\sum_{F \in \mathcal{F}_{T}} h_{F}^{-1}\left(\boldsymbol{\pi}_{F}^{k}\left(\boldsymbol{v}_{F}-\hat{\boldsymbol{r}}_{T}^{k+1}\left(\underline{\boldsymbol{v}}_{T}\right)\right), \boldsymbol{\pi}_{F}^{k}\left(\boldsymbol{w}_{F}-\hat{\boldsymbol{r}}_{T}^{k+1}\left(\underline{\boldsymbol{w}}_{T}\right)\right)\right)_{F},
$$

where $\hat{\boldsymbol{r}}_{T}^{k+1}: \underline{\boldsymbol{U}}_{T}^{k} \rightarrow \mathbb{P}_{k+1}(T)^{d}$ denotes a second velocity reconstruction operator defined by adding to $\boldsymbol{v}_{T}$ a high-order correction inferred from $\boldsymbol{r}_{T}^{k+1}\left(\underline{\boldsymbol{v}}_{T}\right)$,

$$
\hat{\boldsymbol{r}}_{T}^{k+1}\left(\underline{\boldsymbol{v}}_{T}\right):=\boldsymbol{v}_{T}+\left(\boldsymbol{r}_{T}^{k+1}\left(\underline{\boldsymbol{v}}_{T}\right)-\boldsymbol{\pi}_{T}^{k} \boldsymbol{r}_{T}^{k+1}\left(\underline{\boldsymbol{v}}_{T}\right)\right) \text {. }
$$

This (rather subtle) choice ensures that the penalty term leads to the stability and boundedness result stated in (27) below, while at the same time preserving the optimal approximation properties of the potential reconstruction $\boldsymbol{r}_{T}^{k+1}$, so that, for all $\boldsymbol{v} \in \boldsymbol{H}^{k+2}(T)$,

$$
s_{T}\left(\underline{\boldsymbol{I}}_{T}^{k} \boldsymbol{v}, \underline{\boldsymbol{I}}_{T}^{k} \boldsymbol{v}\right)^{1 / 2} \lesssim h_{T}^{k+1}\|\boldsymbol{v}\|_{\boldsymbol{H}^{k+2}(T)} .
$$

Remark 1 (Variations). Following [9], we observe that it is possible to modify the local velocity space as follows:

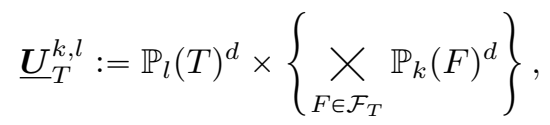


for $k \geqslant 0$ and $l \in\{k-1, k, k+1\}$, so that the case $l=k$ corresponds to the approach presented above. The local velocity reconstruction operator $\boldsymbol{r}_{T}^{k+1}$ still maps onto $\mathbb{P}_{k+1}(T)^{d}$ and is defined by (5) (only its domain changes, but we keep the same notation for simplicity). The discrete bilinear form $a_{T}$ is still defined by (7), but the stabilization bilinear form $s_{T}$ now uses the velocity reconstruction operator $\hat{\boldsymbol{r}}_{T}^{k+1, l}$ such that $\hat{\boldsymbol{r}}_{T}^{k+1, l}\left(\underline{\boldsymbol{v}}_{T}\right):=\boldsymbol{v}_{T}+\left(\boldsymbol{r}_{T}^{k+1}\left(\underline{\boldsymbol{v}}_{T}\right)-\boldsymbol{\pi}_{T}^{l} \boldsymbol{r}_{T}^{k+1}\left(\underline{\boldsymbol{v}}_{T}\right)\right)$ for all $\underline{\boldsymbol{v}}_{T} \in \underline{\boldsymbol{U}}_{T}^{k, l}$. The advantage of the choice $l=k+1$ is that it leads to a particularly simple expression for $s_{T}$, namely

$$
s_{T}\left(\underline{\boldsymbol{v}}_{T}, \underline{\boldsymbol{w}}_{T}\right)=\sum_{F \in \mathcal{F}_{T}} h_{F}^{-1}\left(\boldsymbol{\pi}_{F}^{k}\left(\boldsymbol{v}_{F}-\boldsymbol{v}_{T}\right), \boldsymbol{\pi}_{F}^{k}\left(\boldsymbol{w}_{F}-\boldsymbol{w}_{T}\right)\right)_{F}
$$

(Note that this choice for $s_{T}$ is not appropriate for $l=k$ since it does not deliver the high-order consistency property (10).) The interest in the choice $l=k-1$ is that the discretization of the viscous term is then closely related to the recent High-Order Mimetic method studied in [33] for diffusive problems. Moreover, in the case $k=0$ and $l=-1$, we recover the Crouzeix-Raviart discretization of the viscous term considered in [30], provided the following conventions are adopted: (i) $\mathbb{P}_{-1}(T)^{d}=\{\mathbf{0}\}$, so that cell velocities are not needed; (ii) the local velocity reconstruction operator $\boldsymbol{r}_{T}^{1}$ is defined such that $\boldsymbol{r}_{T}^{1}\left(\underline{\boldsymbol{v}}_{T}\right)=\tilde{\boldsymbol{v}}_{T}$ where $\tilde{\boldsymbol{v}}_{T}$ is the unique function in $\mathbb{P}_{1}(T)^{d}$ such that $\int_{F} \tilde{\boldsymbol{v}}_{T}=\int_{F} \boldsymbol{v}_{F}$ for all F $\in \mathcal{F}_{T}$. With these choices, it is readily seen that the stabilization bilinear form vanishes.

\subsection{Pressure-velocity coupling}

We next introduce the main ingredient to realize the pressure-velocity coupling. The discrete pressure space is

$$
P_{T}^{k}:=\mathbb{P}_{k}(T)
$$

Following [16], we define the discrete divergence operator $D_{T}^{k}: \underline{U}_{T}^{k} \rightarrow P_{T}^{k}$ as follows: For a given local collection of velocities $\underline{\boldsymbol{v}}_{T} \in \underline{\boldsymbol{U}}_{T}^{k}, D_{T}^{k}\left(\underline{\boldsymbol{v}}_{T}\right)$ is such that, for all $q \in \mathbb{P}_{k}(T)$,

$$
\begin{aligned}
\left(D_{T}^{k}\left(\underline{\boldsymbol{v}}_{T}\right), q\right)_{T} & =\left(\operatorname{div}\left(\boldsymbol{v}_{T}\right), q\right)_{T}+\sum_{F \in \mathcal{F}_{T}}\left(\boldsymbol{v}_{F}-\boldsymbol{v}_{T}, q \boldsymbol{n}_{T F}\right)_{F} \\
& =-\left(\boldsymbol{v}_{T}, \boldsymbol{\nabla} q\right)_{T}+\sum_{F \in \mathcal{F}_{T}}\left(\boldsymbol{v}_{F}, q \boldsymbol{n}_{T F}\right)_{F}
\end{aligned}
$$

Crucially, this operator satisfies the commuting property

$$
D_{T}^{k}\left(\underline{\boldsymbol{I}}_{T}^{k} \boldsymbol{v}\right)=\pi_{T}^{k}(\operatorname{div} \boldsymbol{v}) \quad \forall \boldsymbol{v} \in \boldsymbol{H}^{1}(T) .
$$

The pressure-velocity coupling is realized by means of the bilinear form $b_{T}$ on $\underline{U}_{T}^{k} \times P_{T}^{k}$ such that, for all $\left(\underline{\boldsymbol{v}}_{T}, q_{T}\right) \in \underline{\boldsymbol{U}}_{T}^{k} \times P_{T}^{k}$,

$$
b_{T}\left(\underline{\boldsymbol{v}}_{T}, q_{T}\right):=-\left(D_{T}^{k}\left(\underline{\boldsymbol{v}}_{T}\right), q_{T}\right) \text {. }
$$

\subsection{Divergence-preserving velocity reconstruction}

The ingredients discussed in the previous sections are sufficient to devise a well-posed discrete problem leading to optimal error bounds with respect to the mesh-size. To achieve robustness of the error estimates with respect to small diffusion, however, a new ingredient is needed whose goal is to yield an exact balance of the curl-free part in the discrete momentum equation. Such a balance is formulated at the continuous level by testing the momentum equation against a divergence-free velocity test function. At the discrete level, this property is obtained by using in the discretization of the body forces a local, divergence-preserving velocity reconstruction operator whose normal component at mesh interfaces only depends on the face-based discrete velocities. Let $T \in \mathcal{T}_{h}$ and let $\mathbb{R T}_{k}(T)$ denote the Raviart-Thomas polynomial space of degree $k$ on $T$. We define the operator $\boldsymbol{R}_{T}^{k}: \underline{U}_{T}^{k} \rightarrow \mathbb{R T}_{k}(T)$ such that, for all $\underline{\boldsymbol{v}}_{T} \in \underline{\boldsymbol{U}}_{T}^{k}$,

$$
\begin{aligned}
\left(\boldsymbol{R}_{T}^{k}\left(\underline{\boldsymbol{v}}_{T}\right), \boldsymbol{w}\right)_{T} & =\left(\boldsymbol{v}_{T}, \boldsymbol{w}\right)_{T}, & & \forall \boldsymbol{w} \in \mathbb{P}_{k-1}(T)^{d}, \\
\left(\boldsymbol{R}_{T}^{k}\left(\underline{\boldsymbol{v}}_{T}\right) \cdot \boldsymbol{n}_{T F}, q\right)_{F} & =\left(\boldsymbol{v}_{F} \cdot \boldsymbol{n}_{T F}, q\right)_{F}, & & \forall q \in \mathbb{P}_{k}(F), \quad \forall F \in \mathcal{F}_{T},
\end{aligned}
$$


where it is understood that $(16 \mathrm{a})$ is not needed in the lowest-order case $k=0$. The key properties of $\boldsymbol{R}_{T}^{k}$ are summarized in the following lemma.

Lemma 2 (Properties of $\boldsymbol{R}_{T}^{k}$ ). The operator $\boldsymbol{R}_{T}^{k}$ is divergence-preserving, i.e., for all $\underline{\boldsymbol{v}}_{T} \in \underline{\boldsymbol{U}}_{T}^{k}$, the following holds:

$$
\operatorname{div}\left(\boldsymbol{R}_{T}^{k}\left(\underline{\boldsymbol{v}}_{T}\right)\right)=D_{T}^{k}\left(\underline{\boldsymbol{v}}_{T}\right),
$$

and $\boldsymbol{R}_{T}^{k}\left(\underline{\boldsymbol{v}}_{T}\right)_{\mid F} \cdot \boldsymbol{n}_{T F}$ only depends on $\boldsymbol{v}_{F} \cdot \boldsymbol{n}_{T F}$. Additionally, for all $\underline{\boldsymbol{v}}_{T} \in \underline{\boldsymbol{U}}_{T}^{k}$, the following bound holds:

$$
\left\|\boldsymbol{R}_{T}^{k}\left(\underline{\boldsymbol{v}}_{T}\right)-\boldsymbol{v}_{T}\right\|_{T} \lesssim \sum_{F \in \mathcal{F}_{T}} h_{F}^{1 / 2}\left\|\left(\boldsymbol{v}_{T}-\boldsymbol{v}_{F}\right) \cdot \boldsymbol{n}_{T F}\right\|_{F} .
$$

Proof. For all $q \in \mathbb{P}_{k}(T)$, we observe that

$$
\begin{aligned}
\left(\operatorname{div}\left(\boldsymbol{R}_{T}^{k}\left(\underline{\boldsymbol{v}}_{T}\right)\right), q\right)_{T} & =-\left(\boldsymbol{R}_{T}^{k}\left(\underline{\boldsymbol{v}}_{T}\right), \boldsymbol{\nabla} q\right)_{T}+\sum_{F \in \mathcal{F}_{T}}\left(\boldsymbol{R}_{T}^{k}\left(\underline{\boldsymbol{v}}_{T}\right) \cdot \boldsymbol{n}_{T F}, q\right)_{F} \\
& =-\left(\boldsymbol{v}_{T}, \boldsymbol{\nabla} q\right)_{T}+\sum_{F \in \mathcal{F}_{T}}\left(\boldsymbol{v}_{F} \cdot \boldsymbol{n}_{T F}, q\right)_{F}=\left(D_{T}^{k}\left(\underline{\boldsymbol{v}}_{T}\right), q\right)_{T},
\end{aligned}
$$

where we have used integration by parts together with the definitions (16) and (13) of $\boldsymbol{R}_{T}^{k}$ and $D_{T}^{k}$, respectively. This proves (17) since both $\operatorname{div}\left(\boldsymbol{R}_{T}^{k}\left(\underline{\boldsymbol{v}}_{T}\right)\right)$ and $D_{T}^{k}\left(\underline{\boldsymbol{v}}_{T}\right)$ are in $\mathbb{P}_{k}(T)$. The fact that $\boldsymbol{R}_{T}^{k}\left(\underline{\boldsymbol{v}}_{T}\right)_{\mid F} \cdot \boldsymbol{n}_{T F}$ only depends on $\boldsymbol{v}_{F} \cdot \boldsymbol{n}_{T F}$ results from classical properties of the polynomial space $\mathbb{R}_{k}(T)$. Finally, we apply to $\boldsymbol{\varphi}_{T}=\boldsymbol{R}_{T}^{k}\left(\underline{\boldsymbol{v}}_{T}\right)-\boldsymbol{v}_{T}$ the estimate

$$
\left\|\boldsymbol{\varphi}_{T}\right\|_{T} \lesssim \sup _{\boldsymbol{w} \in \mathbb{P}_{k-1}(T)^{d},\|\boldsymbol{w}\|_{T} \leqslant 1}\left(\boldsymbol{\varphi}_{T}, \boldsymbol{w}\right)_{T}+\sum_{F \in \mathcal{F}_{T}} h_{F}^{1 / 2}\left\|\boldsymbol{\varphi}_{T} \cdot \boldsymbol{n}_{T F}\right\|_{F},
$$

which is valid for any function $\varphi_{T} \in \mathbb{R} \mathbb{T}_{k}(T)$, and obtain (18) since the sup-term is zero. Estimate (19) is proved using the Piola transformation to the reference element and the basis representation of an arbitrary $\varphi_{T} \in \mathbb{R T}_{k}(T)$, where the coefficients are integral moments of $\boldsymbol{\varphi}_{T}$ over cell $T$ and integral moments of $\boldsymbol{\varphi}_{T} \cdot \boldsymbol{n}_{T F}$ over the faces $F \in \mathcal{F}_{T}$ (see [8, p. 549-555]).

\subsection{Assembly of the global problem}

The global discrete velocity space $\underline{\boldsymbol{U}}_{h}^{k}$ is obtained by prescribing the single-valuedness of interface unknowns so that, if $F \in \mathcal{F}_{h}^{\mathrm{i}}$ is a common face of two elements $T_{1}, T_{2} \in \mathcal{T}_{h}$, then the approximations $\boldsymbol{v}_{F}$ in the collections $\underline{\boldsymbol{v}}_{T_{1}}$ and $\underline{\boldsymbol{v}}_{T_{2}}$ coincide,

$$
\underline{\boldsymbol{U}}_{h}^{k}:=\left\{\underline{\boldsymbol{v}}_{h}=\left(\left(\boldsymbol{v}_{T}\right)_{T \in \mathcal{T}_{h}},\left(\boldsymbol{v}_{F}\right)_{F \in \mathcal{F}_{h}}\right) \mid \underline{\boldsymbol{v}}_{T}:=\left(\boldsymbol{v}_{T},\left(\boldsymbol{v}_{F}\right)_{F \in \mathcal{F}_{T}}\right) \in \underline{\boldsymbol{U}}_{T}^{k} \quad \forall T \in \mathcal{T}_{h}\right\} .
$$

For all $\underline{\boldsymbol{v}}_{h} \in \underline{\boldsymbol{U}}_{h}^{k}$ and all $F \in \mathcal{F}_{h}^{\mathrm{i}}$, the single-valuedness of $\boldsymbol{v}_{F}$ across interfaces ensures that the field $\boldsymbol{w}$ such that $\boldsymbol{w}_{\mid T}=\boldsymbol{R}_{T}^{k}\left(\underline{\boldsymbol{v}}_{T}\right)$ for all $T \in \mathcal{T}_{h}$ is in $\boldsymbol{H}(\operatorname{div} ; \Omega)$. To enforce the homogeneous Dirichlet boundary condition, we consider the following subspace of $\underline{\boldsymbol{U}}_{h}^{k}$ :

$$
\underline{\boldsymbol{U}}_{h, 0}^{k}:=\left\{\underline{\boldsymbol{v}}_{h} \in \underline{\boldsymbol{U}}_{h}^{k} \mid \boldsymbol{v}_{F} \equiv \mathbf{0} \quad \forall F \in \mathcal{F}_{h}^{\mathrm{b}}\right\}
$$

Furthermore, the discrete pressure space is defined to be

$$
P_{h}^{k}:=\mathbb{P}_{k}\left(\mathcal{T}_{h}\right), \quad P_{h, 0}^{k}:=P_{h}^{k} \cap L_{0}^{2}(\Omega),
$$

with the broken polynomial space $\mathbb{P}_{k}\left(\mathcal{T}_{h}\right):=\left\{q_{h} \in L^{2}(\Omega) \mid q_{T}:=q_{h \mid T} \in \mathbb{P}_{k}(T), \forall T \in \mathcal{T}_{h}\right\}$.

Global bilinear forms $a_{h}$ and $b_{h}$ on $\underline{\boldsymbol{U}}_{h}^{k} \times \underline{\boldsymbol{U}}_{h}^{k}$ and $\underline{\boldsymbol{U}}_{h}^{k} \times P_{h}^{k}$, respectively, are obtained by assembling the local contributions element-wise as follows:

$$
a_{h}\left(\underline{\boldsymbol{v}}_{h}, \underline{\boldsymbol{w}}_{h}\right):=\sum_{T \in \mathcal{T}_{h}} a_{T}\left(\underline{\boldsymbol{v}}_{T}, \underline{\boldsymbol{w}}_{T}\right), \quad b_{h}\left(\underline{\boldsymbol{v}}_{h}, q_{h}\right):=\sum_{T \in \mathcal{T}_{h}} b_{T}\left(\underline{\boldsymbol{v}}_{T}, q_{T}\right),
$$


with $a_{T}$ defined by (7) and $b_{T}$ by (15). The discrete problem reads: Find $\left(\underline{\boldsymbol{u}}_{h}, p_{h}\right) \in \underline{\boldsymbol{U}}_{h, 0}^{k} \times P_{h, 0}^{k}$ such that,

$$
\begin{aligned}
\nu a_{h}\left(\underline{\boldsymbol{u}}_{h}, \underline{\boldsymbol{v}}_{h}\right)+b_{h}\left(\underline{\boldsymbol{v}}_{h}, p_{h}\right) & =\ell_{h}\left(\underline{\boldsymbol{v}}_{h}\right) & & \forall \underline{\boldsymbol{v}}_{h} \in \underline{\boldsymbol{U}}_{h, 0}^{k}, \\
b_{h}\left(\underline{\boldsymbol{u}}_{h}, q_{h}\right) & =0 & & \forall q_{h} \in P_{h, 0}^{k},
\end{aligned}
$$

with right-hand side of the discrete momentum equation such that

$$
\ell_{h}\left(\underline{\boldsymbol{v}}_{h}\right):=\sum_{T \in \mathcal{T}_{h}}\left(\boldsymbol{f}, \boldsymbol{R}_{T}^{k}\left(\underline{\boldsymbol{v}}_{T}\right)\right)_{T} .
$$

\section{Main results}

In this section, we state our main results and refer to Section 7 for the proofs.

\subsection{Stability}

We introduce the following discrete $\boldsymbol{H}^{1}$-like semi-norm on $\underline{\boldsymbol{U}}_{h}^{k}$ :

$$
\left\|\underline{\boldsymbol{v}}_{h}\right\|_{1, h}^{2}:=\sum_{T \in \mathcal{T}_{h}}\left\|\underline{\boldsymbol{v}}_{T}\right\|_{1, T}^{2}, \quad\left\|\underline{\boldsymbol{v}}_{T}\right\|_{1, T}^{2}:=\left\|\boldsymbol{\nabla} \boldsymbol{v}_{T}\right\|_{T}^{2}+\sum_{F \in \mathcal{F}_{T}} h_{F}^{-1}\left\|\boldsymbol{v}_{F}-\boldsymbol{v}_{T}\right\|_{F}^{2} \quad \forall T \in \mathcal{T}_{h} .
$$

A direct verification shows that the map $\|\cdot\|_{1, h}$ defines a norm on the space $\underline{\boldsymbol{U}}_{h, 0}^{k}$ defined by (21). The stability properties of the bilinear forms $a_{h}$ and $b_{h}$ and the well-posedness of the discrete problem (24) are summarized in the following Lemma.

Lemma 3 (Stability and well-posedness). There is a real number $\eta>0$, independent of $h$, such that, for all $\underline{\boldsymbol{v}}_{h} \in \underline{\boldsymbol{U}}_{h}^{k}$,

$$
\eta\left\|\underline{\boldsymbol{v}}_{h}\right\|_{1, h}^{2} \leqslant a_{h}\left(\underline{\boldsymbol{v}}_{h}, \underline{\boldsymbol{v}}_{h}\right) \leqslant \eta^{-1}\left\|\underline{\boldsymbol{v}}_{h}\right\|_{1, h}^{2} .
$$

Additionally, the following holds for all $q_{h} \in P_{h, 0}^{k}$ :

$$
\beta\left\|q_{h}\right\| \leqslant \sup _{\underline{\boldsymbol{v}}_{h} \in \underline{\boldsymbol{U}}_{h, 0}^{k},\left\|\underline{\boldsymbol{v}}_{h}\right\|_{1, h} \leqslant 1} b_{h}\left(\underline{\boldsymbol{v}}_{h}, q_{h}\right),
$$

with real number $\beta>0$ independent of $h$. Finally, problem (24) is well-posed.

Proof. See Section 7.1.

\subsection{Convergence}

We collect in this section the main results concerning the convergence analysis of problem (24). We start by an energy error estimate which yields a robust bound for the velocity in the small viscosity limit. Define the global velocity reduction operator $\underline{\boldsymbol{I}}_{h}^{k}: \boldsymbol{H}^{1}(\Omega) \rightarrow \underline{\boldsymbol{U}}_{h}^{k}$ such that, for all $\boldsymbol{v} \in \boldsymbol{H}^{1}(\Omega)$ (cf. (4) for the definition of $\underline{\boldsymbol{I}}_{T}^{k}$ ):

$$
\left(\underline{\boldsymbol{I}}_{h}^{k} \boldsymbol{v}\right)_{T}=\underline{\boldsymbol{I}}_{T}^{k}\left(\boldsymbol{v}_{\mid T}\right), \quad \forall T \in \mathcal{T}_{h} .
$$

Our goal is to bound the discrete velocity error $\underline{\boldsymbol{u}}_{h}-\underline{\boldsymbol{I}}_{h}^{k}(\boldsymbol{u}) \in \underline{\boldsymbol{U}}_{h, 0}^{k}$ and the discrete pressure error $p_{h}-\pi_{h}^{k} p \in P_{h, 0}^{k}$ where $\pi_{h}^{k}$ denotes the $L^{2}$-orthogonal projector onto $P_{h}^{k}$.

Theorem 4 (Convergence rate). Let $(\boldsymbol{u}, p) \in \boldsymbol{H}_{0}^{1}(\Omega) \times L_{0}^{2}(\Omega)$ and $\left(\underline{\boldsymbol{u}}_{h}, p_{h}\right) \in \underline{\boldsymbol{U}}_{h, 0}^{k} \times P_{h, 0}^{k}$ denote the unique solutions of (2) and (24), respectively, and assume the additional regularity

$$
\boldsymbol{u} \in \boldsymbol{H}^{k+2}(\Omega) .
$$

Then, the following holds:

$$
\begin{aligned}
\left\|\underline{\boldsymbol{u}}_{h}-\underline{\boldsymbol{I}}_{h}^{k} \boldsymbol{u}\right\|_{1, h} & \lesssim h^{k+1}\|\boldsymbol{u}\|_{\boldsymbol{H}^{k+2}(\Omega)} \\
\beta\left\|p_{h}-\pi_{h}^{k} p\right\| & \lesssim \nu h^{k+1}\|\boldsymbol{u}\|_{\boldsymbol{H}^{k+2}(\Omega)} .
\end{aligned}
$$


Proof. See Section 7.2

Remark 5 (Role of $\boldsymbol{R}_{T}^{k}$ ). The use of the divergence-preserving velocity reconstruction operator $\boldsymbol{R}_{T}^{k}$ in the right-hand side of the discrete problem (24) is crucial to achieve the error estimate of Theorem 4. Using in (24a) the standard choice

$$
\ell_{h}\left(\underline{\boldsymbol{v}}_{h}\right)=\sum_{T \in \mathcal{T}_{h}}\left(\boldsymbol{f}, \boldsymbol{v}_{T}\right)_{T},
$$

instead of (25), and under the additional regularity assumption $p \in H^{k+1}(\Omega)$, we obtain the following bound on the velocity error:

$$
\left\|\underline{\boldsymbol{u}}_{h}-\underline{\boldsymbol{I}}_{h}^{k} \boldsymbol{u}\right\|_{1, h} \lesssim h^{k+1}\left(\|\boldsymbol{u}\|_{\boldsymbol{H}^{k+2}(\Omega)}+\nu^{-1}\|p\|_{H^{k+1}(\Omega)}\right) .
$$

The term $\nu^{-1}\|p\|_{H^{k+1}(\Omega)}$ in the right-hand side grows unboundedly when $\nu \rightarrow 0^{+}$, therefore compromising the accuracy of the velocity approximation. We refer to Remark 11 for further insight concerning the proof of (33).

Remark 6 (Estimate for the pressure error). Under the additional regularity assumption $p \in H^{k+1}(\Omega)$, we infer from (31) the following bound on the pressure error:

$$
\beta\left\|p_{h}-p\right\| \lesssim \nu h^{k+1}\|\boldsymbol{u}\|_{\boldsymbol{H}^{k+2}(\Omega)}+\beta h^{k+1}\|p\|_{H^{k+1}(\Omega)} .
$$

The second result stated in this section is an optimally-convergent, viscosity-independent $L^{2}$-velocity error estimate under the following elliptic regularity assumption: There is $c_{\text {ell }}$, depending only on $\Omega$, such that, for all $\boldsymbol{g} \in \boldsymbol{L}^{2}(\Omega)$, the unique solution of the Stokes problem

$$
\begin{array}{rlr}
-\nu \triangle \boldsymbol{z}+\nabla \theta=\boldsymbol{g} & & \text { in } \Omega, \\
\operatorname{div} \boldsymbol{z}=0 & & \text { in } \Omega, \\
\boldsymbol{z}=\mathbf{0} & & \text { on } \Gamma,
\end{array}
$$

satisfies the regularity estimate

$$
\nu\|\boldsymbol{z}\|_{\boldsymbol{H}^{2}(\Omega)}+\|\theta\|_{H^{1}(\Omega)} \leqslant c_{\mathrm{ell}}\|\boldsymbol{g}\|_{\boldsymbol{L}^{2}(\Omega)} .
$$

This estimate holds, for instance, if the domain $\Omega$ is convex; cf. [6, 2].

Theorem 7 ( $L^{2}$-velocity error estimate). Under the assumptions of Theorem 4 and the above elliptic regularity assumption, the following holds:

$$
\left\|\boldsymbol{e}_{h}\right\|_{\boldsymbol{L}^{2}(\Omega)} \lesssim h^{k+2}\|\boldsymbol{u}\|_{\boldsymbol{H}^{k+2}(\Omega)},
$$

where $\boldsymbol{e}_{h} \in \boldsymbol{L}^{2}(\Omega)$ is such that $\boldsymbol{e}_{h \mid T}=\boldsymbol{u}_{T}-\boldsymbol{\pi}_{T}^{k} \boldsymbol{u}$ for all $T \in \mathcal{T}_{h}$. Additionally, letting $\breve{\boldsymbol{u}}_{h} \in \mathbb{P}_{k+1}\left(\mathcal{T}_{h}\right)^{d}$ be such that $\breve{\boldsymbol{u}}_{h \mid T}=\boldsymbol{r}_{T}^{k+1}\left(\underline{\boldsymbol{u}}_{T}\right)$ for all $T \in \mathcal{T}_{h}$, we have

$$
\left\|\breve{\boldsymbol{u}}_{h}-\boldsymbol{u}\right\| \lesssim h^{k+2}\|\boldsymbol{u}\|_{\boldsymbol{H}^{k+2}(\Omega)} .
$$

The bound (37) shows that supercloseness holds for the discrete cell velocities (we have convergence at order $(k+2)$ despite using polynomials of degree $k$ ). This is, more generally, a salient feature of HHO methods; cf. the details and a comparison with HDG in [9]. The estimate (38) shows the practical advantage that can be taken from supercloseness. By a computationally cheap postprocessing step, we can compute the discontinuous approximation $\breve{\boldsymbol{u}}_{h}$ of polynomial degree $(k+1)$ in each element which guarantees the order $(k+2)$ in the $L^{2}$-norm. If we would apply a second averaging postprocessing step to $\breve{\boldsymbol{u}}_{h}$, we could also compute a conforming approximation of order $(k+1)$ which consists elementwise of polynomials of the same degree. 


\subsection{Local conservation}

We follow the general ideas of [15] to identify the local conservation properties of the method; see also [9]. To uncover the numerical fluxes, the first step consists in post-processing the discrete velocities inside each element. Let $T \in \mathcal{T}_{h}$ and define the local bilinear form on $\underline{\boldsymbol{U}}_{T}^{k} \times \underline{\boldsymbol{U}}_{T}^{k}$ such that

$$
\tilde{a}_{T}\left(\underline{\boldsymbol{v}}_{T}, \underline{\boldsymbol{w}}_{T}\right):=\left(\boldsymbol{\nabla} \boldsymbol{r}_{T}^{k+1}\left(\underline{\boldsymbol{v}}_{T}\right), \boldsymbol{\nabla} \boldsymbol{r}_{T}^{k+1}\left(\underline{\boldsymbol{w}}_{T}\right)\right)_{T}+j_{T}\left(\underline{\boldsymbol{v}}_{T}, \underline{\boldsymbol{w}}_{T}\right)
$$

with $\boldsymbol{r}_{T}^{k+1}$ defined by (5) and

$$
j_{T}\left(\underline{\boldsymbol{v}}_{T}, \underline{\boldsymbol{w}}_{T}\right):=\sum_{F \in \mathcal{F}_{T}} h_{F}^{-1}\left(\boldsymbol{v}_{F}-\boldsymbol{v}_{T}, \boldsymbol{w}_{F}-\boldsymbol{w}_{T}\right)_{F}
$$

where the sole difference with respect to the local bilinear form $a_{T}$ defined by (7) lies in the stabilization term. We define the isomorphism $\underline{\boldsymbol{c}}_{T}^{k}: \underline{\boldsymbol{U}}_{T}^{k} \rightarrow \underline{\boldsymbol{U}}_{T}^{k}$ such that, for a given $\underline{\boldsymbol{v}}_{T} \in \underline{\boldsymbol{U}}_{T}^{k}, \underline{\boldsymbol{c}}_{T}^{k}\left(\underline{\boldsymbol{v}}_{T}\right)$ is the unique solution of

$$
\tilde{a}_{T}\left(\underline{\boldsymbol{c}}_{T}^{k}\left(\underline{\boldsymbol{v}}_{T}\right), \underline{\boldsymbol{w}}_{T}\right)=a_{T}\left(\underline{\boldsymbol{v}}_{T}, \underline{\boldsymbol{w}}_{T}\right)+j_{T}\left(\underline{\boldsymbol{v}}_{T}, \underline{\boldsymbol{w}}_{T}\right) \quad \forall \underline{\boldsymbol{w}}_{T} \in \underline{\boldsymbol{U}}_{T}^{k},
$$

with closure condition $\int_{T}\left(\underline{\boldsymbol{c}}_{T}^{k}\left(\underline{\boldsymbol{v}}_{T}\right)\right)_{T}=\int_{T} \boldsymbol{v}_{T}$. We then define the post-processed local velocity by means of the operator $\tilde{\boldsymbol{r}}_{T}^{k+1}: \underline{\boldsymbol{U}}_{T}^{k} \rightarrow \mathbb{P}_{k+1}(T)^{d}$ such that

$$
\tilde{\boldsymbol{r}}_{T}^{k+1}:=\boldsymbol{r}_{T}^{k+1} \circ \underline{\boldsymbol{c}}_{T}^{k} .
$$

Because of the use of the divergence-preserving velocity reconstruction operator $\boldsymbol{R}_{T}^{k}$ in the right-hand side of the discrete problem, for all $T \in \mathcal{T}_{h}$ and all $F \in \mathcal{F}_{T}$ we introduce an additional lifting operator $\boldsymbol{L}_{T F}^{k}: \boldsymbol{L}^{2}(T) \rightarrow \mathbb{P}_{k}(F)^{d}$ satisfying, for all $\boldsymbol{g} \in \boldsymbol{L}^{2}(T)$,

$$
\left(\boldsymbol{L}_{T F}^{k}(\boldsymbol{g}), \boldsymbol{w}\right)_{F}=\left(\boldsymbol{g}, \boldsymbol{R}_{T}^{k}\left(\underline{\boldsymbol{\epsilon}}_{T F}(\boldsymbol{w})\right)\right)_{T}, \quad \forall \boldsymbol{w} \in \mathbb{P}_{k}(F)^{d},
$$

where $\underline{\epsilon}_{T F}(\boldsymbol{w}) \in \underline{\boldsymbol{U}}_{T}^{k}$ is defined such that $\left(\underline{\boldsymbol{\epsilon}}_{T F}(\boldsymbol{w})\right)_{T}=\mathbf{0},\left(\underline{\boldsymbol{\epsilon}}_{T F}(\boldsymbol{w})\right)_{F^{\prime}}=\mathbf{0}$ for all $F^{\prime} \in \mathcal{F}_{T}$ with $F^{\prime} \neq F$, and $\left(\underline{\epsilon}_{T F}(\boldsymbol{w})\right)_{F}=\boldsymbol{w}$. Finally, the numerical momentum and mass fluxes $\boldsymbol{\Phi}_{T F}^{k}: \underline{\boldsymbol{U}}_{T}^{k} \times \mathbb{P}_{k}(T) \rightarrow \mathbb{P}_{k}(F)^{d}$ and $\phi_{T F}^{k}: \underline{U}_{T}^{k} \rightarrow \mathbb{P}_{k}(F)$ are defined as

$$
\begin{aligned}
\boldsymbol{\Phi}_{T F}^{k}\left(\underline{\boldsymbol{u}}_{T}, p_{T}\right):= & \left(\nu \boldsymbol{\nabla} \tilde{\boldsymbol{r}}_{T}^{k+1}\left(\underline{\boldsymbol{u}}_{T}\right)-p_{T} \mathbf{I}_{d}\right) \boldsymbol{n}_{T F}-\boldsymbol{L}_{T F}^{k}\left(\boldsymbol{f}-\boldsymbol{\pi}_{T}^{k-1} \boldsymbol{f}\right) \\
& +\nu h_{F}^{-1}\left(\left(\underline{\boldsymbol{\delta}}_{T}^{k}\left(\underline{\boldsymbol{u}}_{T}\right)\right)_{F}-\left(\underline{\boldsymbol{\delta}}_{T}^{k}\left(\underline{\boldsymbol{u}}_{T}\right)\right)_{T}\right), \\
\phi_{T F}^{k}\left(\underline{\boldsymbol{u}}_{T}\right):= & \boldsymbol{u}_{F} \cdot \boldsymbol{n}_{T F},
\end{aligned}
$$

with $\underline{\boldsymbol{\delta}}_{T}^{k}\left(\underline{\boldsymbol{u}}_{T}\right):=\underline{\boldsymbol{c}}_{T}^{k}\left(\underline{\boldsymbol{u}}_{T}\right)-\underline{\boldsymbol{u}}_{T}$ and $\mathbf{I}_{d}$ the identity matrix in $\mathbb{R}^{d \times d}$.

Proposition 8 (Local conservation). The numerical fluxes defined in (42) satisfy

$$
\begin{array}{r}
\boldsymbol{\Phi}_{T_{1} F}^{k}\left(\underline{\boldsymbol{u}}_{T_{1}}, p_{T_{1}}\right)+\boldsymbol{\Phi}_{T_{2} F}^{k}\left(\underline{\boldsymbol{u}}_{T_{2}}, p_{T_{2}}\right)=\mathbf{0}, \\
\phi_{T_{1} F}^{k}\left(\underline{\boldsymbol{u}}_{T_{1}}\right)+\phi_{T_{2} F}^{k}\left(\underline{\boldsymbol{u}}_{T_{2}}\right)=0,
\end{array}
$$

and the discrete solution $\left(\underline{\boldsymbol{u}}_{h}, p_{h}\right)$ of $(24)$ satisfies, for all $T \in \mathcal{T}_{h}$, all $\boldsymbol{v}_{T} \in \mathbb{P}_{k}(T)^{d}$, and all $q_{T} \in \mathbb{P}_{k}(T)$,

$$
\begin{gathered}
\left(\nu \nabla \tilde{\boldsymbol{r}}_{T}^{k+1}\left(\underline{\boldsymbol{u}}_{T}\right)-p_{T} \mathbf{I}_{d}, \boldsymbol{\nabla} \boldsymbol{v}_{T}\right)_{T}-\sum_{F \in \mathcal{F}_{T}}\left(\boldsymbol{\Phi}_{T F}^{k}\left(\underline{\boldsymbol{u}}_{T}, p_{T}\right), \boldsymbol{v}_{T}\right)_{F}=\left(\boldsymbol{f}, \boldsymbol{v}_{T}\right)_{T}, \\
\left(\boldsymbol{u}_{T}, \boldsymbol{\nabla} q_{T}\right)_{T}-\sum_{F \in \mathcal{F}_{T}}\left(\phi_{T F}^{k}\left(\underline{\boldsymbol{u}}_{T}\right), q_{T}\right)_{F}=0 .
\end{gathered}
$$

Proof. See Section 7.4

Remark 9 (Lifting $\left.\boldsymbol{L}_{T F}^{k}\right)$. One can easily verify that $\boldsymbol{L}_{T F}^{k}(\boldsymbol{g})$ is collinear to $\boldsymbol{n}_{T F}$. Moreover, provided $\boldsymbol{f}$ is smooth enough, it is readily shown using (18) and the triangle inequality that $\left\|\boldsymbol{L}_{T F}^{k}\left(\boldsymbol{f}-\boldsymbol{\pi}_{T}^{k-1} \boldsymbol{f}\right)\right\|_{F} \lesssim$ $h_{T}^{k+1 / 2}\|\boldsymbol{f}\|_{\boldsymbol{H}^{k}(T)}$. The last term in (42a) depending on $\underline{\boldsymbol{\delta}}_{T}^{k}\left(\underline{\boldsymbol{u}}_{T}\right)$ is of the same order in $h_{T}$. 


\section{$5 \quad$ Numerical results}

In this section, several numerical examples in 2D and in 3D are presented to illustrate the theoretical results achieved. The numerical results are intended to emphasize the qualitative difference between the classical discretization of the right-hand side term (32) and the novel discretization (25). The results are presented for three different discrete norms. The discrete velocity and pressure errors are defined as

$$
\underline{\boldsymbol{e}}_{\boldsymbol{u}, h}:=\underline{\boldsymbol{u}}_{h}-\underline{\boldsymbol{I}}_{h}^{k} \boldsymbol{u}, \quad e_{p, h}:=p_{h}-\pi_{h}^{k} p .
$$

The velocity error is measured in the norm

$$
\left\|\underline{\boldsymbol{e}}_{\boldsymbol{u}, h}\right\|_{a, h}:=a_{h}\left(\underline{\boldsymbol{e}}_{\boldsymbol{u}, h}, \underline{\boldsymbol{e}}_{\boldsymbol{u}, h}\right)^{1 / 2},
$$

which corresponds to a discrete version of the velocity error in the $H^{1}$ seminorm; by (27), this norm is equivalent to the $H^{1}$-like norm $\|\cdot\|_{1, h}$ defined by (26). We also measure the velocity error in the usual $L^{2}$-norm. Note that in the $\boldsymbol{L}^{2}(\Omega)$ norm only the cell-based DOFs are taken into account. The pressure error $e_{p, h}$ is measured in the usual $L^{2}$-norm. Unstructured grids are generated using the TRIANGLE library [38] in 2d and the TETGEN library [39] in 3d. The whole implementation is performed in $\mathrm{C}++$ whithin the PDELIB framework [40].

The 2D and 3D examples in Subsection 5.1 illustrate that the classical discretization of the right-hand side forcing $\boldsymbol{f}$ (32) leads to a scheme which is not pressure-robust, while the more sophisticated discretization (25) makes the scheme pressure-robust. Note that the stiffness matrix for both discretizations is the same. The 2D and 3D examples in Subsection 5.2 show that the schemes converge with the theoretical expected orders of convergence, if $\nu$ is fixed to the value 1.0.

\subsection{Pressure-robustness with respect to $\nu$}

2d example First, we look at a two-dimensional Stokes problem in $\Omega=(0,1)^{2}$ with a prescribed academic solution. The stream function of the velocity field is given by

$$
\xi=x^{2}(x-1)^{2} y^{2}(y-1)^{2} .
$$

Then, for $(\boldsymbol{u}, p)=\left(\operatorname{curl} \xi, x^{5}+y^{5}-\frac{1}{3}\right)$, we obtain $\boldsymbol{u} \in \boldsymbol{H}_{0}^{1}(\Omega), \nabla \cdot \boldsymbol{u}=0$ and $p \in L_{0}^{2}(\Omega)$, see Figure 2 .

The right-hand side is computed from the solution by setting $\boldsymbol{f}:=-\nu \Delta \boldsymbol{u}+\nabla p$, and it is clear that $\boldsymbol{f} \in \boldsymbol{L}^{2}(\Omega)$. For $\nu \rightarrow 0$, we have that $\|\boldsymbol{f}\|_{\boldsymbol{L}^{2}(\Omega)} \rightarrow\|\nabla p\|_{\boldsymbol{L}^{2}(\Omega)}$, and this quantity remains bounded and converges to a fixed value. In Figure 1 numerical results for the velocity error $\left\|\underline{e}_{\boldsymbol{u}, h}\right\|_{a, h}$ and the pressure error $\left\|e_{p, h}\right\|$ are presented on the coarsest level with 30 triangles for the polynomial orders $k=0,1,2$ and $\nu=1,10^{-1}, 10^{-2}, 10^{-3}$, varying also the discretization of the right-hand side according to (32) (referred to as "classical") and (25) (referred to as "pressure-robust"). The discrete velocity error in the corresponding $\boldsymbol{L}^{2}$-norm is not shown, since it behaves with respect to $\nu$ in a completely analogous manner as $\left\|\boldsymbol{e}_{\boldsymbol{u}, h}\right\|_{a, h}$. The velocity error $\left\|\boldsymbol{e}_{\boldsymbol{u}, h}\right\|_{a, h}$ deteriorates for $\nu \rightarrow 0$, and is asymptotically proportional to $\frac{1}{\nu}$ as predicted by the theory. This illustrates that the discrete velocities of mixed finite element methods with the classical right-hand side discretization (32) are not pressure-robust. Indeed, the discrete velocity error can be arbitrarily distant to the velocity best approximation, even if the data remains bounded. On general unstructured grids, this probem can be avoided only if the continuous pressure $p$ lies accidentally in the discrete pressure space. However, in the above example, the prescribed fifth-order pressure does not lie in the discrete pressure space for $k=0,1,2$. Last but not least, the discrete pressure error is regarded. Contrary to the discrete velocity error, the discrete pressure error for the discretization (32) behaves in a robust manner for $\nu \rightarrow 0$. However, the right-hand discretization (25) delivers not only a discrete pressure error, which is robust, but even converges to 0 for $\nu \rightarrow 0$ ! Therefore, the novel discretization (25) improves both velocity and pressure simultaneously.

3d example The second example illustrates the $\nu$-dependence and $\nu$-independence of the discretizations with (32) and (25), respectively, in the 3D case on a fixed unstructured grid with 360 tetrahedra. 


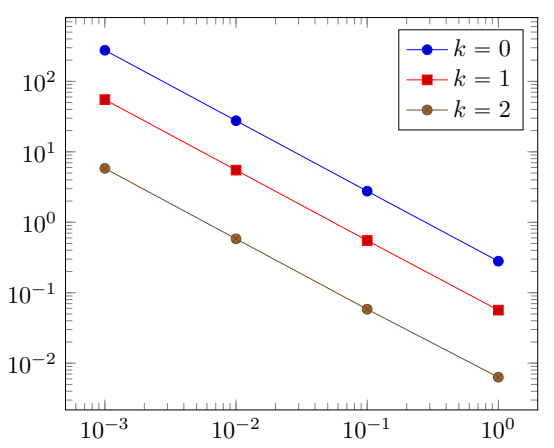

(a) $\left\|\underline{e}_{u, h}\right\|_{a, h}$ vs. $\nu$, classical

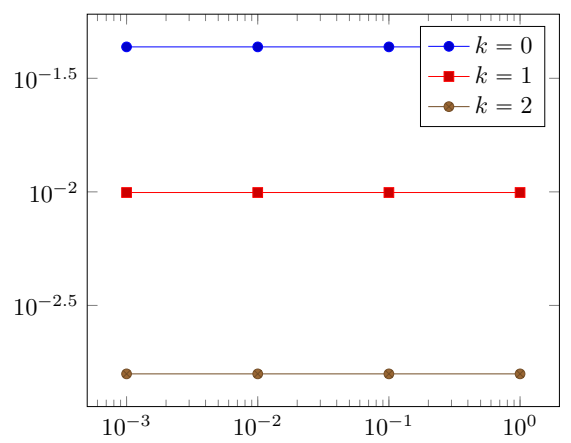

(c) $\left\|\underline{\boldsymbol{e}}_{\boldsymbol{u}, h}\right\|_{a, h}$ vs. $\nu$, pressure-robust

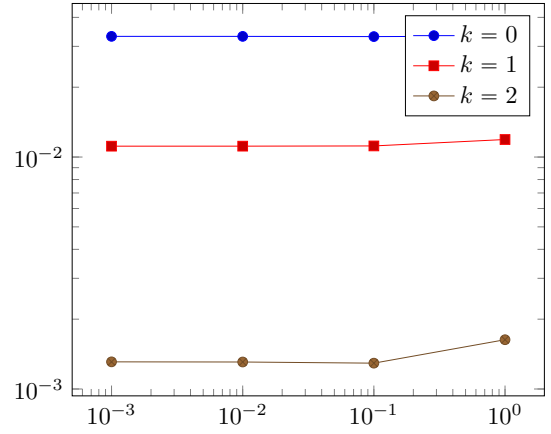

(b) $\left\|e_{p, h}\right\|$ vs. $\nu$, classical

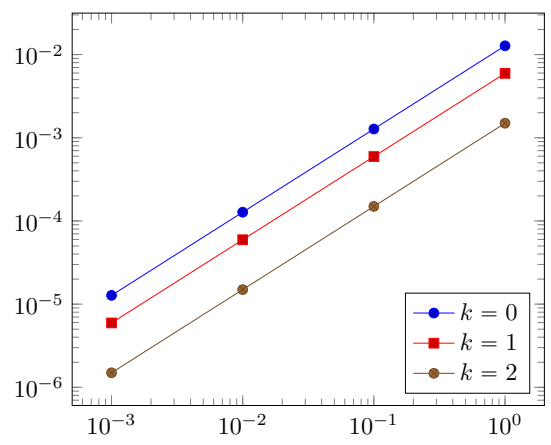

(d) $\left\|e_{p, h}\right\|$ vs. $\nu$, pressure-robust

Figure 1: Results for the 2d example of Section 5.1.

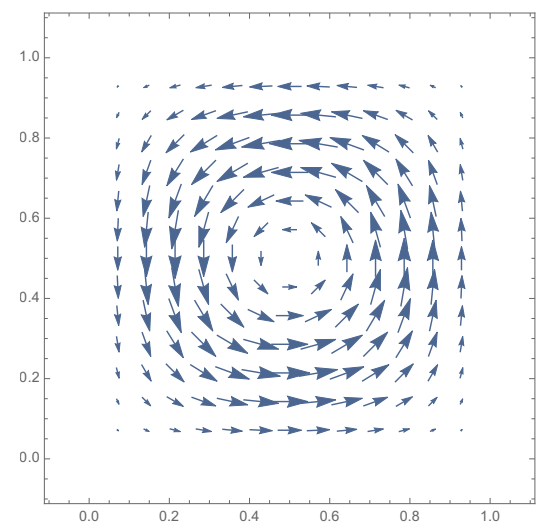

Figure 2: Flow field from 2d example in Section 5.1

For the velocity, a fifth-order polynomial Stokes potential flow is investigated in $\Omega=(0,1)^{3}$, i.e., $\boldsymbol{u}=\nabla h$ with

$$
h=5 x^{6}-90 x^{4} y^{2}+120 x^{2} y^{4}-16 y^{6}+15 x^{4} z^{2}-180 x^{2} y^{2} z^{2}+120 y^{4} z^{2}+15 x^{2} z^{4}-90 y^{2} z^{4}+5 z^{6},
$$

where $h$ is harmonic, and also a fifth-order polynomial is prescribed for the pressure as $p=x^{5}+y^{5}+z^{5}-\frac{1}{2}$. The velocity field is sketched in Figure 4. This example is slightly more difficult than the previous one, and it is nearer to computational practice, since now the Dirichlet boundary conditions are inhomogeneous and do not lie in the discrete velocity space. Therefore, the complete $\nu$-independence of (25) may not be possible theoretically. However, in practice (25) behaves nearly in a $\nu$-robust manner and (32) is lacking robustness as in the $2 \mathrm{D}$ example, see Figure 3. Therefore, again (25) behaves much better than the classical discretization (32). 


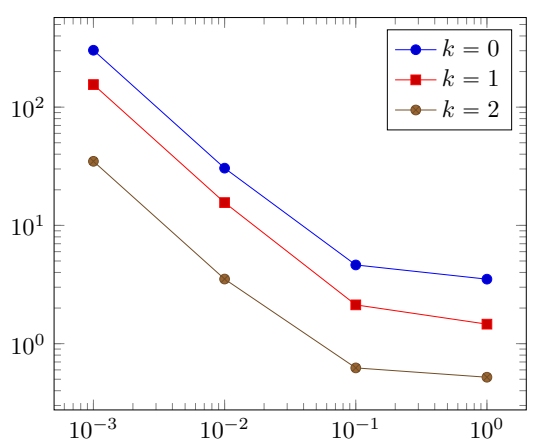

(a) $\left\|\underline{e}_{u, h}\right\|_{a, h}$ vs. $\nu$, classical

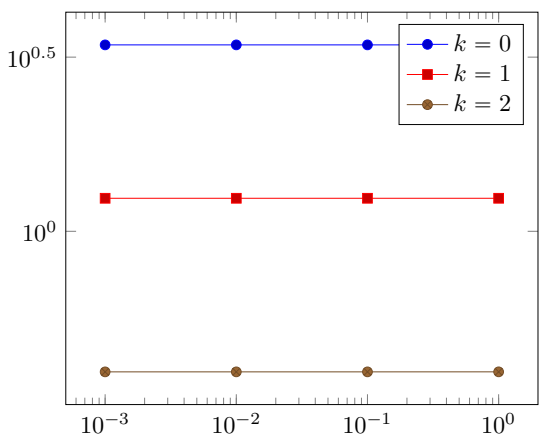

(c) $\left\|\underline{\boldsymbol{e}}_{\boldsymbol{u}, h}\right\|_{a, h}$ vs. $\nu$, pressure-robust

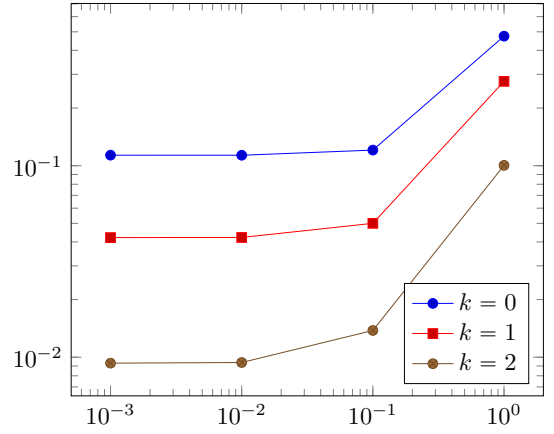

(b) $\left\|e_{p, h}\right\|$ vs. $\nu$, classical

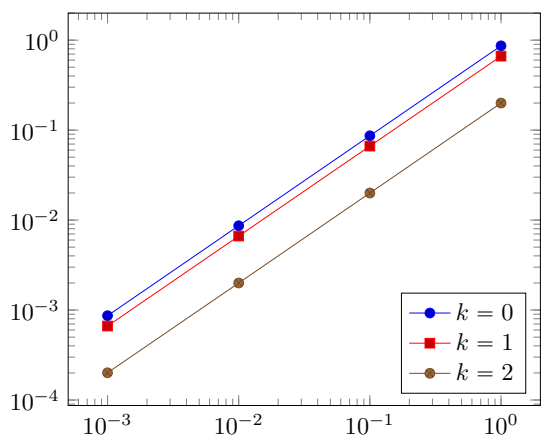

(d) $\left\|e_{p, h}\right\|$ vs. $\nu$, pressure-robust

Figure 3: Results for the 3d example of Section 5.1.

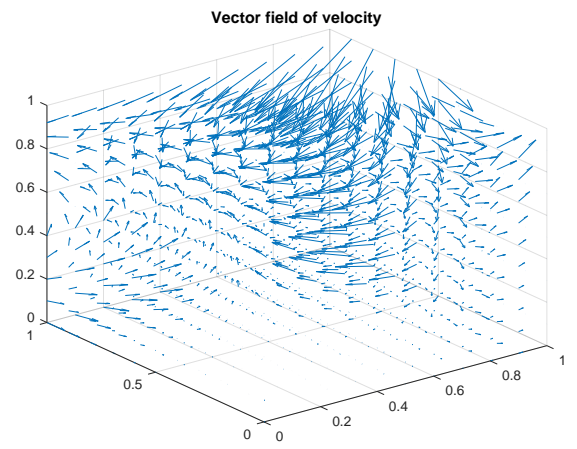

Figure 4: Flow field from 3d example in Section 5.1

\subsection{Convergence}

2d example To assess the convergence rates, we repeat the numerical $2 \mathrm{~d}$ example of the previous section, but now the viscosity $\nu$ is fixed to 1 and the mesh size and the polynomial orders $k=0,1,2$ of the approximation spaces are varied. This example serves as an illustration that all the theoretically predicted convergence rates are indeed met by both 2D discretizations (32) and (25). For the numerical results see Table 1. 


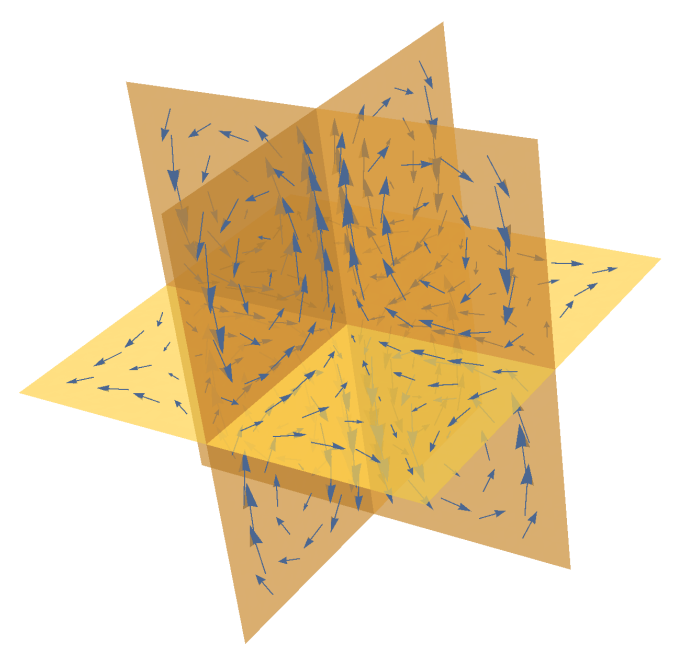

Figure 5: Flow field from 3d example in Section 5.2

3d example The last numerical example is intended to show the expected convergence rates on a sequence of unstructured tetrahedral meshes for $k=0,1,2$ and for both discretizations in the 3D case. Moreover, now the example is not polynomial. This especially means that the right-hand side terms are approximated by numerical quadratures. For the velocity $\boldsymbol{u}=\left(u_{1}, u_{2}, u_{3}\right)^{T}$ in $\Omega=(0,1)^{3}$ the components $u_{1}=\frac{1}{2} \sin (2 \pi x) \cos (2 \pi y) \cos (2 \pi z), u_{2}=\frac{1}{2} \cos (2 \pi x) \sin (2 \pi y) \cos (2 \pi z)$, and $u_{3}=-\cos (2 \pi x) \cos (2 \pi y) \sin (2 \pi z)$ are prescribed, see Figure 5. The pressure is set to $p=\sin (2 \pi x) \sin (2 \pi y) \sin (2 \pi z)$. The numerical results in Table 2 show that the theoretically expected convergence rates are recovered.

\section{Computational aspects}

We can reduce substantially the total number of degrees of freedom (DOFs) if we eliminate by static condensation those local unknows on each mesh cell $T \in \mathcal{T}_{h}$ that are coupled only to unknowns associated with the cell $T$. For the solution $\underline{\boldsymbol{u}}_{h}=\left(\left(\boldsymbol{u}_{T}\right)_{T \in \mathcal{T}_{h}},\left(\boldsymbol{u}_{F}\right)_{F \in \mathcal{F}_{h}}\right)$ and $p_{h}=\left(p_{T}\right)_{T \in \mathcal{T}_{h}}$, the local unknowns that can be eliminated on $T \in \mathcal{T}_{h}$ are the cell-based DOFs associated with $\boldsymbol{u}_{T}$ and those associated with the component of $p_{T}$ in the subspace $L_{0}^{2}(T):=\left\{q \in L^{2}(T) ;(q, 1)_{T}=0\right\}$. The unknowns that remain in the assembled reduced global system are those associated with the face velocities $\left(\boldsymbol{u}_{F}\right)_{F \in \mathcal{F}_{h}^{\mathbf{i}}}$ (boundary faces $F \in \mathcal{F}_{h}^{\mathrm{b}}$ are omitted since the wall boundary condition (1c) is strongly enforced) and the constant pressure mean values $\bar{p}_{0}^{T}:=|T|^{-1}\left(p_{T}, 1\right)_{T}$. In what follows, we describe in detail the static condensation process from an algebraic viewpoint.

\subsection{Expansions of discrete variables}

Let $T \in \mathcal{T}_{h}$ be a given mesh cell and let $\underline{\boldsymbol{U}}_{T}^{k}$ be the $T$-local velocity space defined in (3). To create a basis of $\underline{\boldsymbol{U}}_{T}^{k}$, we choose in a first step a basis of $\mathbb{P}_{k}(T)^{d}$, i.e.,

$$
\mathbb{P}_{k}(T)^{d}=\operatorname{span}\left\{\varphi_{j}^{T}, j \in \hat{J}_{T}^{v}\right\}, \quad \hat{J}_{T}^{v}:=\left\{1, \ldots, \operatorname{dim}\left(\mathbb{P}_{k}(T)^{d}\right)\right\},
$$

and define the associated basis functions $\underline{\varphi}^{T} \in \underline{\boldsymbol{U}}_{T}^{k}$ as

$$
\underline{\varphi}_{j}^{T}:=\left(\boldsymbol{\varphi}_{j}^{T},(\mathbf{0})_{F \in \mathcal{F}_{T}}\right) \quad \forall j \in \hat{J}_{T}^{v} .
$$


In a second step, we define the basis functions associated with the interior faces of $\partial T$. Let $F_{m}, m=$ $1, \ldots, M_{T}$, denote the interior faces of the set $\mathcal{F}_{T}$. Then, we choose basis functions of $\mathbb{P}_{k}\left(F_{m}\right)^{d}$, i.e.

$$
\mathbb{P}_{k}\left(F_{m}\right)^{d}=\operatorname{span}\left\{\varphi_{j}^{F_{m}}, j \in \hat{J}_{F_{m}}^{v}\right\}, \quad \hat{J}_{F_{m}}^{v}:=\left\{1, \ldots, \operatorname{dim}\left(\mathbb{P}_{k}\left(F_{m}\right)^{d}\right)\right\}
$$

and define the associated basis functions $\underline{\varphi}_{j}^{F_{m}} \in \underline{\boldsymbol{U}}_{T}^{k}$ as

$$
\underline{\varphi}_{j}^{F_{m}}:=\left(\mathbf{0},\left(\varphi_{F}\right)_{F \in \mathcal{F}_{T}}\right) \quad \text { with } \quad \boldsymbol{\varphi}_{F_{m}}=\varphi_{j}^{F_{m}}, \boldsymbol{\varphi}_{F}=\mathbf{0} \quad \forall F \in \mathcal{F}_{T} \backslash\left\{F_{m}\right\} .
$$

We collect all face basis functions $\underline{\varphi}_{j}^{F_{m}}, j \in \hat{J}_{F_{m}}^{v}, m=1, \ldots, M_{T}$, into one set of basis fuctions $\underline{\varphi}_{j}^{\partial T}$, $j \in \hat{J}_{\partial T}^{v}:=\bigcup_{m=1}^{M_{T}} \hat{J}_{\partial T, m}^{v}$, where $\hat{J}_{\partial T, m}^{v}:=\hat{n}_{m}^{T}+\hat{J}_{F_{m}}^{v}=\left\{\hat{n}_{m}^{T}+1, \ldots, \hat{n}_{m}^{T}+\operatorname{dim}\left(\mathbb{P}_{k}\left(F_{m}\right)^{d}\right)\right\}$ denote the shifted local index sets associated with the faces $F_{m}$ which are disjoint due to the shift parameters $\hat{n}_{m}^{T}$ defined recursively by $\hat{n}_{1}^{T}:=0$ and $\hat{n}_{i+1}^{T}:=\hat{n}_{i}^{T}+\operatorname{dim}\left(\mathbb{P}_{k}\left(F_{i}\right)^{d}\right)$ for $i=1, \ldots M_{T}-1$. Therefore, each index $j \in \hat{J}_{\partial T}^{v}$ belongs to exactly one face $F_{m}$ in the sense that there exists a unique $m$ so that $j \in \hat{J}_{\partial T, m}^{v}$, and we define $\underline{\varphi}_{j}^{\partial T}:=\underline{\boldsymbol{\varphi}}_{j-\hat{n}_{m}^{T}}^{F_{m}}$. Now we can write each $\underline{\boldsymbol{u}}_{T} \in \underline{\boldsymbol{U}}_{T}^{k}$ in the basis representation

$$
\underline{\boldsymbol{u}}_{T}=\sum_{j \in \hat{J}_{T}^{v}} \bar{u}_{j}^{T, \ell} \underline{\boldsymbol{\varphi}}_{j}^{T}+\sum_{j \in \hat{J}_{\partial T}^{v}} \bar{u}_{j}^{T, g} \underline{\boldsymbol{\varphi}}_{j}^{\partial T},
$$

where $\bar{u}_{j}^{T, \ell}$ denote the local scalar DOFs which can be eliminated on cell $T$ and $\bar{u}_{j}^{T, g}$ the global scalar DOFs which remain in the reduced global system of equations.

Let us now consider the $T$-local pressure space $P_{T}^{k}:=\mathbb{P}_{k}(T)$. At first, we choose as one basis function $\psi_{0}^{T} \in P_{T}^{k}$ the characteristic function $\psi_{0}^{T}(x):=1$ for $\boldsymbol{x} \in T$. The rest of the local basis functions we choose from the subspace $P_{T}^{k} \cap L_{0}^{2}(T)$ such that

$$
P_{T}^{k} \cap L_{0}^{2}(T)=\operatorname{span}\left\{\psi_{j}^{T}, j \in \hat{J}_{T}^{p}\right\}, \quad \hat{J}_{T}^{p}:=\left\{1, \ldots, \operatorname{dim}\left(\mathbb{P}_{k}(T)\right)-1\right\} .
$$

Now we can write each $p_{T} \in P_{T}^{k}$ in the basis representation

$$
p_{T}=\sum_{j \in \hat{J}_{T}^{p}} \bar{p}_{j}^{T, \ell} \psi_{j}^{T}+\bar{p}^{T, g} \psi_{0}^{T},
$$

where $\bar{p}_{j}^{T, \ell}$ denote the local scalar DOFs which can be eliminated on cell $T$ and $\bar{p}^{T, g}$ denotes the one global scalar DOF from $T$ which remains in the reduced global system. By construction the DOF $\bar{p}^{T, g}$ is the integral mean value $\bar{p}^{T, g}=|T|^{-1}\left(p_{T}, 1\right)_{T}$.

\subsection{Static condensation}

We can now describe how the local DOFs on cell $T$ are eliminated. Owing to the basis representations (45) and (46), we introduce the local vectors $\bar{u}^{T, \ell}=\left(\bar{u}_{j}^{T, \ell}\right)_{j \in \hat{J}_{T}^{v}}, \bar{u}^{T, g}=\left(\bar{u}_{j}^{T, g}\right)_{j \in \hat{J}_{\partial T}^{v}}$ and $\bar{p}^{T, \ell}=\left(\bar{p}_{j}^{T, \ell}\right)_{j \in \hat{J}_{T}^{p}}$ and define the local matrices

$$
\begin{array}{ll}
\left(A_{T}^{\ell, \ell}\right)_{i, j}:=a_{T}\left(\underline{\varphi}_{j}^{T}, \underline{\varphi}_{i}^{T}\right), i, j \in \hat{J}_{T}^{v}, & \left(A_{T}^{\ell, g}\right)_{i, j}:=a_{T}\left(\underline{\varphi}_{j}^{\partial T}, \underline{\varphi}_{i}^{T}\right), i \in \hat{J}_{T}^{v}, j \in \hat{J}_{\partial T}^{v}, \\
\left(B_{T}^{\ell, \ell}\right)_{i, j}:=b_{T}\left(\underline{\varphi}_{j}^{T}, \psi_{i}^{T}\right), i \in \hat{J}_{T}^{p}, j \in \hat{J}_{T}^{v}, & \left(B_{T}^{\ell, g}\right)_{i, j}:=b_{T}\left(\underline{\varphi}_{j}^{\partial T}, \psi_{i}^{T}\right), i \in \hat{J}_{T}^{p}, j \in \hat{J}_{\partial T}^{v},
\end{array}
$$

as well as the local right-hand side vector $\bar{f}^{T, \ell}=\left(\bar{f}_{j}^{T, \ell}\right)_{j \in \hat{J}_{T}^{v}}$ with $\bar{f}_{j}^{T, \ell}:=\left(\boldsymbol{f}, \boldsymbol{R}_{T}^{k}\left(\underline{\boldsymbol{\varphi}}_{j}^{T}\right)\right)_{T}$. Then, we choose in the first equation (24a) of the discrete problem the test functions $\underline{\boldsymbol{v}}_{h}=\underline{\varphi}_{i}^{T}, i \in \hat{J}_{T}^{v}$, and in the second equation $(24 \mathrm{~b})$ the test functions $q_{h}=\psi_{i}^{T}, i \in \hat{J}_{T}^{p}$, and obtain the following local system for the local vectors $\bar{u}^{T, \ell}$ and $\bar{p}^{T, \ell}$ :

$$
\underbrace{\left(\begin{array}{cc}
\nu A_{T}^{\ell, \ell} & \left(B_{T}^{\ell, \ell}\right)^{\prime} \\
B_{T}^{\ell, \ell} & 0
\end{array}\right)}_{L_{T}^{\ell, \ell}} \underbrace{\left(\begin{array}{c}
\bar{u}^{T, \ell} \\
\bar{p}^{T, \ell}
\end{array}\right)}_{\bar{y}^{T, \ell}}=\underbrace{\left(\begin{array}{c}
\bar{f}^{T, \ell} \\
0
\end{array}\right)}_{\bar{r}^{T, \ell}}-\underbrace{\left(\begin{array}{cc}
\nu A_{T}^{\ell, g} & 0 \\
B_{T}^{\ell, g} & 0
\end{array}\right)}_{L_{T}^{\ell, g}} \underbrace{\left(\begin{array}{c}
\bar{u}^{T, g} \\
\bar{p}^{T, g}
\end{array}\right)}_{\bar{y}^{T, g}} .
$$


Note that the upper right zero block in the matrix $L_{T}^{\ell, g}$ is due to the fact that, for the constant basis function $\psi_{0}^{T}$, we obtain $b_{T}\left(\underline{\varphi}_{i}^{T}, \psi_{0}^{T}\right)=0$ for all $i \in \hat{J}_{T}^{v}$. In Lemma 10 below, we prove that the matrix $L_{T}^{\ell, \ell}$ is non-singular. Therefore, the local unknowns collected in the vector $\bar{y}^{T, \ell}$ can be eliminated from the global system of equations and expressed by the $T$-local global unknowns collected in the vector $\bar{y}^{T, g}$ as

$$
\bar{y}^{T, \ell}=\left(L_{T}^{\ell, \ell}\right)^{-1} \bar{r}^{T, \ell}-\left(L_{T}^{\ell, \ell}\right)^{-1} L_{T}^{\ell, g} \bar{y}^{T, g} .
$$

For an efficient implementation, it is advantageous to store for each mesh cell $T \in \mathcal{T}_{h}$ the local vector $\left(L_{T}^{\ell, \ell}\right)^{-1} \bar{r}^{T, \ell}$ and the local matrix $\left(L_{T}^{\ell, \ell}\right)^{-1} L_{T}^{\ell, g}$. Then, once the reduced global system has been solved, the local vectors $\bar{y}^{T, \ell}$ can be computed very fast in a post-processing loop over all $T \in \mathcal{T}_{h}$.

Lemma 10 (Existence and uniqueness for system (47)). The matrix $L_{T}^{\ell, \ell}$ in the local system (47) is non-singular for each mesh cell $T \in \mathcal{T}_{h}$.

Proof. At first, we show that the (symmetric) block matrix $A_{T}^{\ell, \ell}$ is positive-definite. Let $\bar{u}^{T, \ell}=\left(\bar{u}_{j}^{T, \ell}\right)_{j \in \hat{J}_{T}^{v}}$ be a given vector, and set $\underline{\boldsymbol{u}}_{T}:=\sum_{j \in \hat{J}_{T}^{v}} \bar{u}_{j}^{T, \ell} \underline{\boldsymbol{\varphi}}_{j}^{T} \in \underline{\boldsymbol{U}}_{T}^{k}$. Recalling (27), we infer that $\left(\bar{u}^{T, \ell}\right)^{\prime} A_{T}^{\ell, \ell} \bar{u}^{T, \ell}=$ $a_{T}\left(\underline{\boldsymbol{u}}_{T}, \underline{\boldsymbol{u}}_{T}\right) \geqslant \eta\left\|\underline{\boldsymbol{u}}_{T}\right\|_{1, T}^{2}$. Assuming now that $\left(\bar{u}^{T, \ell}\right)^{\prime} A_{T}^{\ell, \ell} \bar{u}^{T, \ell}=0$ leads to

$$
0=\left\|\underline{\boldsymbol{u}}_{T}\right\|_{1, T}^{2}=\left\|\boldsymbol{\nabla} \boldsymbol{u}_{T}\right\|_{T}^{2}+\sum_{F \in \mathcal{F}_{T}} h_{F}^{-1}\left\|\boldsymbol{u}_{T}\right\|_{F}^{2},
$$

where we have used the fact that the above defined $\underline{\boldsymbol{u}}_{T}=\left(\boldsymbol{u}_{T},\left(\boldsymbol{u}_{F}\right)_{F \in \mathcal{F}_{T}}\right)$ satisfies $\boldsymbol{u}_{F}=\mathbf{0}$ for all $F \in \mathcal{F}_{T}$. From (49) we get $\boldsymbol{\nabla} \boldsymbol{u}_{T}=\mathbf{0}$, i.e. that $\boldsymbol{u}_{T}$ is constant on $T$. Moreover, it follows that $\left.\boldsymbol{u}_{T}\right|_{F}=\mathbf{0}$ for each face $F \in \mathcal{F}_{T}$. Therefore, $\underline{\boldsymbol{u}}_{T}=\mathbf{0}$, which implies that the vector $\bar{u}^{T, \ell}$ is zero. Since $L_{T}^{\ell, \ell}$ has a saddlepoint structure, it only remains to show that the matrix $\left(B_{T}^{\ell, \ell}\right)^{\prime}$ has zero kernel. Assume that the vector $\bar{p}^{T, \ell}=\left(\bar{p}_{j}^{T, \ell}\right)_{j \in \hat{J}_{T}^{p}}$ is in the kernel of $\left(B_{T}^{\ell, \ell}\right)^{\prime}$. Let us define the pressure function

$$
p_{T}:=\sum_{j \in \hat{J}_{T}^{p}} \bar{p}_{j}^{T, \ell} \psi_{j}^{T} \in P_{T}^{k} \cap L_{0}^{2}(T) .
$$

The fact that $\left(B_{T}^{\ell, \ell}\right)^{\prime} \bar{p}^{T, \ell}=0$ implies that $b_{T}\left(\underline{\varphi}_{j}^{T}, p_{T}\right)=\left(\boldsymbol{\varphi}_{j}^{T}, \nabla p_{T}\right)_{T}=0$ for all $j \in \hat{J}_{T}^{v}$. Since $\nabla p_{T} \in$ $\mathbb{P}_{k-1}(T)^{d} \subset \mathbb{P}_{k}(T)^{d}$, we get $\boldsymbol{\nabla} p_{T}=\mathbf{0}$, so that $p_{T}=0$ since $\left(p_{T}, 1\right)_{T}=0$. Hence, $\bar{p}^{T, \ell}$ is zero.

\subsection{Assembly}

Let us finally describe how the reduced global system is assembled by the loop over all mesh cells. Owing to (23), the global stiffness matrix is assembled by adding up, for each mesh cell $T \in \mathcal{T}_{h}$, the local contributions associated with the local bilinear forms $a_{T}(\cdot, \cdot)$ and $b_{T}(\cdot, \cdot)$. The equations in the global system that are affected by contributions from cell $T$ are those where we choose in the first equation (24a) of the discrete problem the test functions $\underline{\boldsymbol{v}}_{h}$ with $\underline{\boldsymbol{v}}_{T}=\underline{\varphi}_{i}^{\partial T}, i \in \hat{J}_{\partial T}^{v}$, and in the second equation (24b) the test function $q_{h}=\psi_{0}^{T}$. We define the local matrices

$$
\begin{array}{ll}
\left(A_{T}^{g, g}\right)_{i, j}:=a_{T}\left(\underline{\varphi}_{j}^{\partial T}, \underline{\varphi}_{i}^{\partial T}\right), i, j \in \hat{J}_{\partial T}^{v}, & \left(A_{T}^{g, \ell}\right)_{i, j}:=a_{T}\left(\underline{\varphi}_{j}^{T}, \underline{\varphi}_{i}^{\partial T}\right), i \in \hat{J}_{\partial T}^{v}, j \in \hat{J}_{T}^{v}, \\
\left(B_{T}^{g, g}\right)_{1, j}:=b_{T}\left(\underline{\varphi}_{j}^{\partial T}, \psi_{0}^{T}\right), j \in \hat{J}_{\partial T}^{v}, & \left(B_{T}^{g, \ell}\right)_{i, j}:=b_{T}\left(\underline{\varphi}_{i}^{\partial T}, \psi_{j}^{T}\right), i \in \hat{J}_{\partial T}^{v}, j \in \hat{J}_{T}^{p},
\end{array}
$$

as well as the local right-hand side vector $\bar{f}^{T, g}=\left(\bar{f}_{j}^{T, g}\right)_{j \in \hat{J}_{\partial T}^{v}}$ with $\bar{f}_{j}^{T, g}:=\left(\boldsymbol{f}, \boldsymbol{R}_{T}^{k}\left(\underline{\boldsymbol{\varphi}}_{j}^{\partial T}\right)\right)_{T}$. Then, the $T$-local block of equations that is added up into the global system is:

$$
\underbrace{\left(\begin{array}{cc}
\nu A_{T}^{g, g} & \left(B_{T}^{g, g}\right)^{\prime} \\
B_{T}^{g, g} & 0
\end{array}\right)}_{L_{T}^{g, g}} \underbrace{\left(\begin{array}{c}
\bar{u}^{T, g} \\
\bar{p}^{T, g}
\end{array}\right)}_{\bar{y}^{T, g}}+\underbrace{\left(\begin{array}{cc}
\nu A_{T}^{g, \ell} & B_{T}^{g, \ell} \\
0 & 0
\end{array}\right)}_{L_{T}^{g, \ell}} \underbrace{\left(\begin{array}{c}
\bar{u}^{T, \ell} \\
\bar{p}^{T, \ell}
\end{array}\right)}_{\bar{y}^{T, \ell}} "=T^{\left(\begin{array}{c}
\bar{f}^{T, g} \\
0
\end{array}\right)}, \underbrace{\underbrace{T, g}}_{\bar{r}^{T, g}}
$$


where the symbol " $=T$ " indicates the principle of adding and storing the local entries into the global system. If we substitute the local vector $\bar{y}^{T, \ell}$ by the elimination formula (48) we get the following $T$-local set of equations that are added and stored into the global system:

$$
\underbrace{\left(L_{T}^{g, g}-L_{T}^{g, \ell}\left(L_{T}^{\ell, \ell}\right)^{-1} L_{T}^{\ell, g}\right)}_{\tilde{L}_{T}^{g, g}} \bar{y}^{T, g} \quad \text { "= } T^{\prime} " \underbrace{\bar{r}^{T, g}-L_{T}^{g, \ell}\left(L_{T}^{\ell, \ell}\right)^{-1} \bar{r}^{T, \ell}}_{\tilde{r}^{T, g}},
$$

i.e., the element stiffness matrix associated with mesh cell $T$ is $\tilde{L}_{T}^{g, g}$ and the element right-hand side vector is $\tilde{r}^{T, g}$.

\section{Proofs}

We collect in this section the proofs of the results stated in Section 4.

\subsection{Proof of Lemma 3}

Proof. See [17, Lemma 4] for (27). For the derivation of (28), we use the inf-sup condition for the continuous problem and infer that there is a constant $\beta_{0}>0$ such that, for each $q_{h} \in P_{h, 0}^{k}$, there exists an element $\boldsymbol{v} \in \boldsymbol{H}_{0}^{1}(\Omega)$ with $\|\boldsymbol{\nabla} \boldsymbol{v}\|_{\Omega} \leqslant 1$ and

$$
\beta_{0}\left\|q_{h}\right\| \leqslant-\sum_{T \in \mathcal{T}_{h}}\left(\operatorname{div} \boldsymbol{v}, q_{h}\right)_{T}
$$

We multiply this inequality by a constant $c_{0}>0$, choose $\underline{\boldsymbol{v}}_{h}:=\underline{\boldsymbol{I}}_{h}^{k}\left(c_{0} \boldsymbol{v}\right)$ and obtain by the commuting property (14)

$$
c_{0} \beta_{0}\left\|q_{h}\right\| \leqslant-\sum_{T \in \mathcal{T}_{h}}\left(D_{T}^{k}\left(\underline{\boldsymbol{I}}_{T}^{k}\left(c_{0} \boldsymbol{v}\right)\right), q_{h}\right)_{T}=b_{h}\left(\underline{\boldsymbol{v}}_{h}, q_{h}\right),
$$

where $c_{0}>0$ will be chosen such that $\left\|\underline{\boldsymbol{v}}_{h}\right\|_{1, h} \leqslant 1$. Thus, the estimate (28) follows with $\beta=c_{0} \beta_{0}$. It remains to show the existence of such an $h$-independent constant $c_{0}$. To this end, we prove that there exists a constant $c_{1}$ such that $\left\|\underline{\boldsymbol{I}}_{h}^{k} \boldsymbol{v}\right\|_{1, h} \leqslant c_{1}\|\boldsymbol{\nabla} \boldsymbol{v}\|_{\Omega}$. Let us denote by $\underline{\boldsymbol{v}}_{T}:=\left(\boldsymbol{\pi}_{T}^{k} \boldsymbol{v},\left(\boldsymbol{\pi}_{F}^{k} \boldsymbol{v}\right)_{F \in \mathcal{F}_{T}}\right)$ the $T$-local part of $\underline{\boldsymbol{I}}_{h}^{k} \boldsymbol{v}$. Then, we get

$$
\left\|\underline{\boldsymbol{v}}_{T}\right\|_{1, T} \lesssim\left\|\boldsymbol{\nabla} \boldsymbol{\pi}_{T}^{k} \boldsymbol{v}\right\|_{T}+\sum_{F \in \mathcal{F}_{T}} h_{F}^{-1 / 2}\left\|\boldsymbol{\pi}_{F}^{k}\left(\boldsymbol{v}-\boldsymbol{\pi}_{T}^{k} \boldsymbol{v}\right)\right\|_{F}:=\mathfrak{T}_{1}+\mathfrak{T}_{2}
$$

Using the $H^{1}$-stability of $\boldsymbol{\pi}_{T}^{k}$ (cf. [14, Lemma 1.58] and [12, Corollary 3.7]), it is readily inferred that $\mathfrak{T}_{1} \lesssim\|\boldsymbol{\nabla} \boldsymbol{v}\|_{T}$. Additionally, combining the stability of $\boldsymbol{\pi}_{F}^{k}$ and the approximation properties of $\boldsymbol{\pi}_{T}^{k}$ proved in [14, Lemma 1.59] (cf. also [12, Lemma 3.4]), one has $\left\|\boldsymbol{\pi}_{F}^{k}\left(\boldsymbol{v}-\boldsymbol{\pi}_{T}^{k} \boldsymbol{v}\right)\right\|_{F} \leqslant\left\|\boldsymbol{v}-\boldsymbol{\pi}_{T}^{k} \boldsymbol{v}\right\|_{F} \lesssim$ $h_{F}^{1 / 2}\|\boldsymbol{\nabla} \boldsymbol{v}\|_{T}$, hence $\mathfrak{T}_{2} \lesssim\|\boldsymbol{\nabla} \boldsymbol{v}\|_{T}$. Plugging the above estimates into (52) shows that there exists an $h$ independent constant $c_{1}>0$ such that $\left\|\underline{\boldsymbol{I}}_{h}^{k} \boldsymbol{v}\right\|_{1, h} \leqslant c_{1}\|\boldsymbol{\nabla} \boldsymbol{v}\|_{\Omega}$ holds. Therefore, the choice $c_{0}:=1 / c_{1}$ leads to $\left\|\underline{\boldsymbol{v}}_{h}\right\|_{1, h}=\left\|\underline{\boldsymbol{I}}_{h}^{k}\left(c_{0} \boldsymbol{v}\right)\right\|_{1, h} \leqslant\|\boldsymbol{\nabla} \boldsymbol{v}\|_{\Omega} \leqslant 1$ for the above function $\boldsymbol{v}$ from the continuous inf-sup condition which concludes the proof of (28). Well-posedness then classically follows from (27) together with (28).

\subsection{Proof of Theorem 4}

Proof of Theorem 4. We define the global discrete divergence operator $D_{h}^{k}: \underline{U}_{h}^{k} \rightarrow P_{h}^{k}$ such that, for all $\underline{\boldsymbol{v}}_{h} \in \underline{\boldsymbol{U}}_{h}^{k}$,

$$
D_{h}^{k}\left(\underline{\boldsymbol{v}}_{h}\right)_{\mid T}=D_{T}^{k}\left(\underline{\boldsymbol{v}}_{T}\right) \quad \forall T \in \mathcal{T}_{h},
$$

and we introduce the space of discretely divergence-free velocities such that

$$
\underline{\boldsymbol{U}}_{h, 0}^{k *}:=\left\{\underline{\boldsymbol{v}}_{h} \in \underline{\boldsymbol{U}}_{h, 0}^{k} \mid D_{h}^{k}\left(\underline{\boldsymbol{v}}_{h}\right)=0\right\} .
$$


Observe that the discrete velocity $\underline{\boldsymbol{u}}_{h}$ resulting from the discrete problem (24) is in $\underline{\boldsymbol{U}}_{h, 0}^{k *}$. We introduce the consistency error

$$
\mathcal{E}_{h}\left(\underline{\boldsymbol{v}}_{h}\right):=\ell_{h}\left(\underline{\boldsymbol{v}}_{h}\right)-\nu a_{h}\left(\underline{\boldsymbol{I}}_{h}^{k} \boldsymbol{u}, \underline{\boldsymbol{v}}_{h}\right)-b_{h}\left(\underline{\boldsymbol{v}}_{h}, \pi_{h}^{k} p\right), \quad \forall \underline{\boldsymbol{v}}_{h} \in \underline{\boldsymbol{U}}_{h, 0}^{k},
$$

and we set

$$
\$:=\sup _{\underline{\boldsymbol{v}}_{h} \in \underline{\boldsymbol{U}}_{h, 0}^{k},\left\|\underline{\boldsymbol{v}}_{h}\right\|_{1, h} \leqslant 1} \mathcal{E}_{h}\left(\underline{\boldsymbol{v}}_{h}\right) .
$$

Owing to the commuting property (14), we infer that $\underline{\boldsymbol{u}}_{h}-\underline{\boldsymbol{I}}_{h}^{k} \boldsymbol{u} \in \underline{\boldsymbol{U}}_{h, 0}^{k *}$. Then, (27) implies that

$$
\nu\left\|\underline{\boldsymbol{u}}_{h}-\underline{\boldsymbol{I}}_{h}^{k} \boldsymbol{u}\right\|_{1, h} \lesssim \sup _{\underline{\boldsymbol{v}}_{h} \in \underline{\boldsymbol{U}}_{h, 0}^{k *},\left\|\underline{\boldsymbol{v}}_{h}\right\|_{1, h} \leqslant 1} \nu a_{h}\left(\underline{\boldsymbol{u}}_{h}-\underline{\boldsymbol{I}}_{h}^{k} \boldsymbol{u}, \underline{\boldsymbol{v}}_{h}\right) .
$$

For all $\underline{\boldsymbol{v}}_{h} \in \underline{\boldsymbol{U}}_{h, 0}^{k *}$, we get by using linearity and (24a),

$$
\begin{aligned}
\nu a_{h}\left(\underline{\boldsymbol{u}}_{h}-\underline{\boldsymbol{I}}_{h}^{k} \boldsymbol{u}, \underline{\boldsymbol{v}}_{h}\right) & =\nu a_{h}\left(\underline{\boldsymbol{u}}_{h}, \underline{\boldsymbol{v}}_{h}\right)-\nu a_{h}\left(\underline{\boldsymbol{I}}_{h}^{k} \boldsymbol{u}, \underline{\boldsymbol{v}}_{h}\right) \\
& =\ell_{h}\left(\underline{\boldsymbol{v}}_{h}\right)-\underbrace{b_{h}\left(\underline{\boldsymbol{v}}_{h}, \pi_{h}^{k} p\right)}_{0}-\nu a_{h}\left(\underline{\boldsymbol{I}}_{h}^{k} \boldsymbol{u}, \underline{\boldsymbol{v}}_{h}\right)=\mathcal{E}_{h}\left(\underline{\boldsymbol{v}}_{h}\right) .
\end{aligned}
$$

Hence, we infer that

$$
\nu\left\|\underline{\boldsymbol{u}}_{h}-\underline{\boldsymbol{I}}_{h}^{k} \boldsymbol{u}\right\|_{1, h} \lesssim \sup _{\underline{\boldsymbol{v}}_{h} \in \underline{\boldsymbol{U}}_{h, 0}^{k *},\left\|\underline{\boldsymbol{v}}_{h}\right\|_{1, h} \leqslant 1} \mathcal{E}_{h}\left(\underline{\boldsymbol{v}}_{h}\right) \leqslant \$ .
$$

Moreover, we observe that the discrete momentum equation (24a) yields

$$
b_{h}\left(\underline{\boldsymbol{v}}_{h}, p_{h}-\pi_{h}^{k} p\right)=\nu a_{h}\left(\underline{\boldsymbol{I}}_{h}^{k} \boldsymbol{u}-\underline{\boldsymbol{u}}_{h}, \underline{\boldsymbol{v}}_{h}\right)+\mathcal{E}_{h}\left(\underline{\boldsymbol{v}}_{h}\right),
$$

for all $\underline{\boldsymbol{v}}_{h} \in \underline{\boldsymbol{U}}_{h, 0}^{k}$. Using the discrete inf-sup condition (28) for $q_{h}=p_{h}-\pi_{h}^{k} p$, the above relation, the Cauchy-Schwarz inequality, and the boundedness of $a_{h}$ expressed by the second inequality in (27), we infer that

$$
\beta\left\|p_{h}-\pi_{h}^{k} p\right\| \leqslant \sup _{\underline{\boldsymbol{v}}_{h} \in \underline{\boldsymbol{U}}_{h, 0}^{k},\left\|\underline{\boldsymbol{v}}_{h}\right\|_{1, h} \leqslant 1} \frac{b_{h}\left(\underline{\boldsymbol{v}}_{h}, p_{h}-\pi_{h}^{k} p\right)}{\left\|\underline{\boldsymbol{v}}_{h}\right\|_{1, h}} \lesssim \nu\left\|\underline{\boldsymbol{u}}_{h}-\underline{\boldsymbol{I}}_{h}^{k} \boldsymbol{u}\right\|_{1, h}+\$ \lesssim \$ .
$$

The above velocity and pressure estimates show that it suffices to bound $\$$ for all $\underline{\boldsymbol{v}}_{h} \in \underline{\boldsymbol{U}}_{h, 0}^{k}$. Using the fact that $\boldsymbol{f}=-\nu \triangle \boldsymbol{u}+\boldsymbol{\nabla} p$ a.e. in $\Omega$, we can decompose the consistency error as follows:

$$
\begin{aligned}
\mathcal{E}_{h}\left(\underline{\boldsymbol{v}}_{h}\right)= & \sum_{T \in \mathcal{T}_{h}} \nu\left\{-\left(\triangle \boldsymbol{u}, \boldsymbol{v}_{T}\right)_{T}-a_{T}\left(\underline{\boldsymbol{I}}_{T}^{k} \boldsymbol{u}, \underline{\boldsymbol{v}}_{T}\right)\right\} \\
& +\sum_{T \in \mathcal{T}_{h}}\left\{\left(\pi_{T}^{k} p, D_{T}^{k} \underline{\boldsymbol{v}}_{T}\right)_{T}+\left(\boldsymbol{\nabla} p, \boldsymbol{R}_{T}^{k}\left(\underline{\boldsymbol{v}}_{T}\right)\right)_{T}\right\} \\
& +\sum_{T \in \mathcal{T}_{h}} \nu\left(\triangle \boldsymbol{u}, \boldsymbol{v}_{T}-\boldsymbol{R}_{T}^{k}\left(\underline{\boldsymbol{v}}_{T}\right)\right)_{T}:=\mathfrak{T}_{1}+\mathfrak{T}_{2}+\mathfrak{T}_{3} .
\end{aligned}
$$

For the first term, integrating by parts the first addend, we obtain

$$
\mathfrak{T}_{1}=\sum_{T \in \mathcal{T}_{h}} \nu\left\{\left(\nabla \boldsymbol{u}, \nabla \boldsymbol{v}_{T}\right)_{T}+\sum_{F \in \mathcal{F}_{T}}\left(\nabla \boldsymbol{u} \cdot \boldsymbol{n}_{T F}, \boldsymbol{v}_{F}-\boldsymbol{v}_{T}\right)_{F}-a_{T}\left(\underline{\boldsymbol{I}}_{T}^{k} \boldsymbol{u}, \underline{\boldsymbol{v}}_{T}\right)\right\},
$$

where, to insert $\boldsymbol{v}_{F}$ in the second term, we have used the regularity assumption (30) on the velocity (in fact that $\boldsymbol{u} \in \boldsymbol{H}^{2}(\Omega)$ ) and the fact that $\boldsymbol{v}_{F}=\mathbf{0}$ on boundary faces. Using the optimal approximation properties of $\left(\boldsymbol{r}_{T}^{k+1} \circ \underline{\boldsymbol{I}}_{T}^{k}\right)$ and $s_{T}$, see (6) and (10), respectively, the term $\boldsymbol{T}_{1}$ can then be estimated as

$$
\left|\mathfrak{T}_{1}\right| \lesssim \nu h^{k+1}\|\boldsymbol{u}\|_{\boldsymbol{H}^{k+2}(\Omega)}\left\|\underline{\boldsymbol{v}}_{h}\right\|_{1, h}
$$

For the term $\mathfrak{T}_{2}$, integrating by parts the second summand and using the fact that $\boldsymbol{R}_{T}^{k}\left(\underline{\boldsymbol{v}}_{T}\right)$ has continuous normal component at mesh interfaces and that $p$ is continuous across interfaces (since the normal components of both $\left(\nu \boldsymbol{\nabla} \boldsymbol{u}-p \mathbf{I}_{d}\right)$ and $\nu \boldsymbol{\nabla} \boldsymbol{u}$ are continuous across interfaces), we infer that

$$
\mathfrak{T}_{2}=\sum_{T \in \mathcal{T}_{h}}\left\{\left(p, D_{T}^{k}\left(\underline{\boldsymbol{v}}_{T}\right)\right)_{T}-\left(p, \operatorname{div}\left(\boldsymbol{R}_{T}^{k}\left(\underline{\boldsymbol{v}}_{T}\right)\right)\right)_{T}\right\}=0
$$


where we have used $D_{T}^{k}\left(\underline{\boldsymbol{v}}_{T}\right) \in \mathbb{P}_{k}(T)$ to replace $\pi_{T}^{k} p$ by $p$ in the first summand and concluded using (17). For the term $\mathfrak{T}_{3}$, using (16a) followed by the Cauchy-Schwarz inequality, mesh regularity, and (18) yields

$$
\mathfrak{T}_{3}=\sum_{T \in \mathcal{T}_{h}} \nu\left(\Delta \boldsymbol{u}-\boldsymbol{\pi}_{T}^{k-1}(\Delta \boldsymbol{u}), \boldsymbol{v}_{T}-\boldsymbol{R}_{T}^{k}\left(\underline{\boldsymbol{v}}_{T}\right)\right)_{T} \lesssim \nu h^{k+1}\|\boldsymbol{u}\|_{\boldsymbol{H}^{k+2}(T)}\left\|\underline{\boldsymbol{v}}_{h}\right\|_{1, h} .
$$

Collecting the above estimates yields the assertion.

Remark 11 (Standard treatment of right-hand side). Using $\ell_{h}\left(\underline{\boldsymbol{v}}_{h}\right)=\sum_{T \in \mathcal{T}_{\boldsymbol{h}}}\left(\boldsymbol{f}, \boldsymbol{v}_{T}\right)_{T}$ instead of (25) in the right-hand side of (24a), the consistency error rewrites as $\mathcal{E}_{h}\left(\underline{\boldsymbol{v}}_{h}\right)=\mathfrak{T}_{1}+\mathfrak{T}_{2}^{\prime}$ with $\mathfrak{T}_{1}$ as above, while

$$
\begin{aligned}
\mathfrak{T}_{2}^{\prime} & =\sum_{T \in \mathcal{T}_{h}}\left\{\left(\pi_{T}^{k} p, D_{T}^{k}\left(\underline{\boldsymbol{v}}_{h}\right)\right)_{T}-\left(\pi_{T}^{k} p, \operatorname{div} \boldsymbol{v}_{T}\right)_{T}-\sum_{F \in \mathcal{F}_{T}}\left(p \boldsymbol{n}_{T F}, \boldsymbol{v}_{F}-\boldsymbol{v}_{T}\right)_{F}\right\} \\
& =\sum_{T \in \mathcal{T}_{h}} \sum_{F \in \mathcal{F}_{T}}\left(\left(\pi_{T}^{k} p-p\right) \boldsymbol{n}_{T F}, \boldsymbol{v}_{F}-\boldsymbol{v}_{T}\right)_{F},
\end{aligned}
$$

where we have used the fact that $\operatorname{div} \boldsymbol{v}_{T} \in \mathbb{P}_{k-1}(T) \subset \mathbb{P}_{k}(T)$ to replace $p$ by $\pi_{T}^{k} p$ in the second summand on the first line and the definition (13a) of $D_{T}^{k}$ in the second line. Hence, assuming the additional regularity $p \in H^{k+1}(\Omega)$, we obtain

$$
\left|\mathfrak{T}_{2}\right| \lesssim h^{k+1}\|p\|_{H^{k+1}(\Omega)}\left\|\underline{\boldsymbol{v}}_{h}\right\|_{1, h},
$$

and this readily leads to the bound (33) on the velocity error.

\subsection{Proof of Theorem 7}

Proof of Theorem \%. Let $(\boldsymbol{z}, \theta)$ be the solution of the Stokes problem (35) with right-hand side $\boldsymbol{e}_{h}$. Set $\underline{\boldsymbol{e}}_{h}:=\underline{\boldsymbol{u}}_{h}-\underline{\boldsymbol{I}}_{h}^{k} \boldsymbol{u}$ and observe that $\boldsymbol{e}_{T}=\boldsymbol{e}_{h \mid T}$ for all $T \in \mathcal{T}_{h}$. A direct calculation shows that

$$
\left\|\boldsymbol{e}_{h}\right\|_{\boldsymbol{L}^{2}(\Omega)}^{2}=\left(\boldsymbol{e}_{h},-\nu \Delta \boldsymbol{z}+\nabla \theta\right)=\mathfrak{T}_{1}+\mathfrak{T}_{2}+\mathfrak{T}_{3},
$$

where

$$
\begin{aligned}
& \mathfrak{T}_{1}:=\sum_{T \in \mathcal{T}_{h}} \nu\left\{\left(\boldsymbol{\nabla} \boldsymbol{e}_{T}, \boldsymbol{\nabla}\left(\boldsymbol{z}-\boldsymbol{r}_{T}^{k+1}\left(\underline{\boldsymbol{I}}_{T}^{k} \boldsymbol{z}\right)\right)_{T}+\sum_{F \in \mathcal{F}_{T}}\left(\boldsymbol{e}_{F}-\boldsymbol{e}_{T}, \boldsymbol{\nabla}\left(\boldsymbol{z}-\boldsymbol{r}_{T}^{k+1}\left(\underline{\boldsymbol{I}}_{T}^{k} \boldsymbol{z}\right)\right) \cdot \boldsymbol{n}_{T F}\right)_{F}\right\},\right. \\
& \mathfrak{T}_{2}:=\sum_{T \in \mathcal{T}_{h}}\left\{-\left(\operatorname{div} \boldsymbol{e}_{T}, \theta\right)_{T}-\sum_{F \in \mathcal{F}_{T}}\left(\boldsymbol{e}_{F}-\boldsymbol{e}_{T}, \theta \boldsymbol{n}_{T F}\right)_{F}\right\}, \\
& \mathfrak{T}_{3}:=\sum_{T \in \mathcal{T}_{h}} \nu\left(\boldsymbol{\nabla} \boldsymbol{r}_{T}^{k+1}\left(\underline{\boldsymbol{u}}_{T}\right)-\boldsymbol{\nabla} \boldsymbol{r}_{T}^{k+1}\left(\underline{\boldsymbol{I}}_{T}^{k} \boldsymbol{u}\right), \boldsymbol{\nabla} \boldsymbol{r}_{T}^{k+1}\left(\underline{\boldsymbol{I}}_{T}^{k} \boldsymbol{z}\right)\right)_{T} .
\end{aligned}
$$

Using (6) (for a function in $\boldsymbol{H}^{2}(T)$ ), it is readily seen that

$$
\left|\mathfrak{T}_{1}\right| \lesssim \nu\left\|\underline{\boldsymbol{e}}_{h}\right\|_{1, h} h\|\boldsymbol{z}\|_{\boldsymbol{H}^{2}(\Omega)} .
$$

Furthermore, since $D_{T}^{k}\left(\underline{\boldsymbol{e}}_{T}\right)=0$, we infer that

$$
\mathfrak{T}_{2}=\sum_{T \in \mathcal{T}_{h}}\left\{-\left(\operatorname{div} \boldsymbol{e}_{T}, \theta-\pi_{T}^{0} \theta\right)_{T}-\sum_{F \in \mathcal{F}_{T}}\left(\boldsymbol{e}_{F}-\boldsymbol{e}_{T},\left(\theta-\pi_{T}^{0} \theta\right) \boldsymbol{n}_{T F}\right)_{F}\right\},
$$

so that

$$
\left|\mathfrak{T}_{2}\right| \lesssim\left\|\underline{\boldsymbol{e}}_{h}\right\|_{1, h} h\|\theta\|_{H^{1}(\Omega)} .
$$

Finally, turning to $\mathfrak{T}_{3}$, we observe that

$$
\mathfrak{T}_{3}=\sum_{T \in \mathcal{T}_{h}}\left\{\left(\boldsymbol{f}, \boldsymbol{R}_{T}^{k}\left(\underline{\boldsymbol{I}}_{T}^{k} \boldsymbol{z}\right)\right)_{T}-b_{T}\left(\underline{\boldsymbol{I}}_{T}^{k} \boldsymbol{z}, p_{T}\right)-\nu s_{T}\left(\underline{\boldsymbol{u}}_{T}, \underline{\boldsymbol{I}}_{T}^{k} \boldsymbol{z}\right)-\nu\left(\nabla \boldsymbol{r}_{T}^{k+1}\left(\underline{\boldsymbol{I}}_{T}^{k} \boldsymbol{u}\right), \boldsymbol{\nabla} \boldsymbol{r}_{T}^{k+1}\left(\underline{\boldsymbol{I}}_{T}^{k} \boldsymbol{z}\right)\right)_{T}\right\} .
$$


Since $D_{T}^{k}\left(\underline{\boldsymbol{I}}_{T}^{k} \boldsymbol{z}\right)=0$, we infer that $\left(\boldsymbol{f}, \boldsymbol{R}_{T}^{k}\left(\underline{\boldsymbol{I}}_{T}^{k} \boldsymbol{z}\right)\right)_{T}=-\nu\left(\triangle \boldsymbol{u}, \boldsymbol{R}_{T}^{k}\left(\underline{\boldsymbol{I}}_{T}^{k} \boldsymbol{z}\right)\right)_{T}$ and that $b_{T}\left(\underline{\boldsymbol{I}}_{T}^{k} \boldsymbol{z}, p_{T}\right)=0$. Re-arranging terms leads to

$$
\mathfrak{T}_{3}=\sum_{T \in \mathcal{T}_{h}} \nu\left\{\left(\boldsymbol{\nabla}\left(\boldsymbol{u}-\boldsymbol{r}_{T}^{k+1}\left(\underline{\boldsymbol{I}}_{T}^{k} \boldsymbol{u}\right)\right), \boldsymbol{\nabla}\left(\boldsymbol{z}-\boldsymbol{r}_{T}^{k+1}\left(\underline{\boldsymbol{I}}_{T}^{k} \boldsymbol{z}\right)\right)\right)_{T}+\left(\triangle \boldsymbol{u}, \boldsymbol{z}-\boldsymbol{R}_{T}^{k}\left(\underline{\boldsymbol{I}}_{T}^{k} \boldsymbol{z}\right)\right)_{T}-s_{T}\left(\underline{\boldsymbol{u}}_{T}, \underline{\boldsymbol{I}}_{T}^{k} \boldsymbol{z}\right)\right\},
$$

whence we infer, proceeding as above, that

$$
\left|\mathfrak{T}_{3}\right| \lesssim \nu\left\|\underline{\boldsymbol{e}}_{h}\right\|_{1, h} h\|\boldsymbol{z}\|_{\boldsymbol{H}^{2}(\Omega)} .
$$

Collecting the above bounds and using the regularity estimate on $\boldsymbol{z}$ yields (37). Let us now show that (38) holds. For all $T \in \mathcal{T}_{h}$, setting $\breve{\boldsymbol{u}}_{T}:=\breve{\boldsymbol{u}}_{h \mid T}=\boldsymbol{r}_{T}^{k+1}\left(\underline{\boldsymbol{u}}_{T}\right)$, we have by application of the triangle inequality

$$
\left\|\breve{\boldsymbol{u}}_{h}-\boldsymbol{u}\right\|^{2}=\sum_{T \in \mathcal{T}_{h}}\left\|\breve{\boldsymbol{u}}_{T}-\boldsymbol{u}\right\|_{T}^{2} \lesssim \sum_{T \in \mathcal{T}_{h}}\left(\left\|\boldsymbol{r}_{T}^{k+1}\left(\underline{\boldsymbol{u}}_{T}-\underline{\boldsymbol{I}}_{T}^{k} \boldsymbol{u}\right)\right\|_{T}^{2}+\left\|\boldsymbol{r}_{T}^{k+1}\left(\underline{\boldsymbol{I}}_{T}^{k} \boldsymbol{u}\right)-\boldsymbol{u}\right\|_{T}^{2}\right):=\mathfrak{T}_{1}+\mathfrak{T}_{2} .
$$

Applying the triangle inequality to the function $\boldsymbol{w}_{T}:=\boldsymbol{r}_{T}^{k+1}\left(\underline{\boldsymbol{u}}_{T}-\underline{\boldsymbol{I}}_{T}^{k} \boldsymbol{u}\right)$ and using for $\left\|\boldsymbol{w}_{T}-\boldsymbol{\pi}_{T}^{0} \boldsymbol{w}_{T}\right\|_{T}$ the local Poincaré inequality for zero-average functions on each mesh cell, we have

$$
\mathfrak{T}_{1} \lesssim \sum_{T \in \mathcal{T}_{h}}\left(h_{T}^{2}\left\|\boldsymbol{\nabla} \boldsymbol{r}_{T}^{k+1}\left(\underline{\boldsymbol{u}}_{T}-\underline{\boldsymbol{I}}_{T}^{k} \boldsymbol{u}\right)\right\|_{T}^{2}+\left\|\boldsymbol{\pi}_{T}^{0}\left(\boldsymbol{u}_{T}-\boldsymbol{\pi}_{T}^{k} \boldsymbol{u}\right)\right\|_{T}^{2}\right) \lesssim h^{k+2}\|\boldsymbol{u}\|_{\boldsymbol{H}^{k+2}(\Omega)},
$$

where the last bound follows using (31a) combined with (27) and the $H^{1}$-stability of $\boldsymbol{r}_{T}^{k+1}$ to estimate the first term in parentheses and the stability of $\boldsymbol{\pi}_{T}^{0}$ followed by (37) to estimate the second. An application of (6) readily yields, on the other hand, $\mathfrak{T}_{2} \lesssim h^{k+2}\|\boldsymbol{u}\|_{\boldsymbol{H}^{k+2}(\Omega)}$, thereby concluding the proof of (38).

\subsection{Proof of Proposition 8}

Proof of Proposition 8. (i) Momentum conservation. Let $T \in \mathcal{T}_{h}$. We infer using the definition (7) of $a_{T}$ followed by (39), (40), and the definition (5) of $\boldsymbol{r}_{T}^{k+1}$, that

$$
\begin{aligned}
a_{T}\left(\underline{\boldsymbol{u}}_{T}, \underline{\boldsymbol{v}}_{T}\right) & =\tilde{a}_{T}\left(\underline{\boldsymbol{c}}_{T}^{k}\left(\underline{\boldsymbol{u}}_{T}\right), \underline{\boldsymbol{v}}_{T}\right)-j_{T}\left(\underline{\boldsymbol{u}}_{T}, \underline{\boldsymbol{v}}_{T}\right) \\
& =\left(\boldsymbol{\nabla} \boldsymbol{r}_{T}^{k+1}\left(\underline{\boldsymbol{c}}_{T}^{k}\left(\underline{\boldsymbol{u}}_{T}\right)\right), \boldsymbol{\nabla} \boldsymbol{r}_{T}^{k+1}\left(\underline{\boldsymbol{v}}_{T}\right)\right)_{T}+j_{T}\left(\underline{\boldsymbol{\delta}}_{T}^{k}\left(\underline{\boldsymbol{u}}_{T}\right), \underline{\boldsymbol{v}}_{T}\right) \\
& =\left(\boldsymbol{\nabla} \tilde{\boldsymbol{r}}_{T}^{k+1}\left(\underline{\boldsymbol{u}}_{T}\right), \boldsymbol{\nabla} \boldsymbol{r}_{T}^{k+1}\left(\underline{\boldsymbol{v}}_{T}\right)\right)_{T}+j_{T}\left(\underline{\boldsymbol{\delta}}_{T}^{k}\left(\underline{\boldsymbol{u}}_{T}\right), \underline{\boldsymbol{v}}_{T}\right) \\
& =\left(\boldsymbol{\nabla} \tilde{\boldsymbol{r}}_{T}^{k+1}\left(\underline{\boldsymbol{u}}_{T}\right), \boldsymbol{\nabla} \boldsymbol{v}_{T}\right)_{T}+\sum_{F \in \mathcal{F}_{T}}\left(\boldsymbol{\nabla} \tilde{\boldsymbol{r}}_{T}^{k+1}\left(\underline{\boldsymbol{u}}_{T}\right) \cdot \boldsymbol{n}_{T F}, \boldsymbol{v}_{F}-\boldsymbol{v}_{T}\right)_{F}+j_{T}\left(\underline{\boldsymbol{\delta}}_{T}^{k}\left(\underline{\boldsymbol{u}}_{T}\right), \underline{\boldsymbol{v}}_{T}\right) .
\end{aligned}
$$

Moreover, owing to (16a), we infer that

$$
\left(\boldsymbol{f}, \boldsymbol{R}_{T}^{k}\left(\underline{\boldsymbol{v}}_{T}\right)\right)_{T}=\left(\boldsymbol{f}-\boldsymbol{\pi}_{T}^{k-1} \boldsymbol{f}, \boldsymbol{R}_{T}^{k}\left(\underline{\boldsymbol{v}}_{T}\right)-\boldsymbol{v}_{T}\right)+\left(\boldsymbol{f}, \boldsymbol{v}_{T}\right)_{T},
$$

and using the definition (16) and the linearity of $\boldsymbol{R}_{T}^{k}$ leads to

$$
\begin{aligned}
\left(\boldsymbol{f}-\boldsymbol{\pi}_{T}^{k-1} \boldsymbol{f}, \boldsymbol{R}_{T}^{k}\left(\underline{\boldsymbol{v}}_{T}\right)-\boldsymbol{v}_{T}\right)_{T} & =\sum_{F \in \mathcal{F}_{T}}\left(\boldsymbol{f}-\boldsymbol{\pi}_{T}^{k-1} \boldsymbol{f}, \boldsymbol{R}_{T}^{k}\left(\underline{\boldsymbol{\epsilon}}_{T F}\left(\boldsymbol{v}_{F}-\boldsymbol{v}_{T \mid F}\right)\right)\right)_{T} \\
& =\sum_{F \in \mathcal{F}_{T}}\left(\boldsymbol{L}_{T F}^{k}\left(\boldsymbol{f}-\boldsymbol{\pi}_{T}^{k-1} \boldsymbol{f}\right), \boldsymbol{v}_{F}-\boldsymbol{v}_{T}\right)_{F}
\end{aligned}
$$

since $\boldsymbol{R}_{T}^{k}\left(\left(\boldsymbol{v}_{T},\left(\boldsymbol{v}_{T \mid F}\right)_{F \in \mathcal{F}_{T}}\right)\right)=\boldsymbol{v}_{T}$. Consider now the discrete momentum equation (24a). Take first a test function $\underline{\boldsymbol{v}}_{h}$ with zero face-based velocities for all $F \in \mathcal{F}_{h}$, and zero cell-based velocities for all $T^{\prime} \in \mathcal{T}_{h}$ with $T^{\prime} \neq T$, while $\boldsymbol{v}_{T}$ is arbitrary in $\mathbb{P}_{k}(T)^{d}$. Recalling that the global bilinear forms $a_{h}$ and $b_{h}$ result from the assembly of local contributions (cf. (23)), plugging the above expressions into (24a), and using the definitions (15) of $b_{T}$ and the definition (13a) of $D_{T}^{k}$ to express $b_{h}\left(\underline{\boldsymbol{u}}_{h}, p_{h}\right)$, equation (44a) follows. Fix now $F \in \mathcal{F}_{h}$ and take a test function $\underline{\boldsymbol{v}}_{h}$ with zero cell-based velocities for all $T \in \mathcal{T}_{h}$, and zero face-based velocities for all $F^{\prime} \in \mathcal{F}_{h}$ with $F^{\prime} \neq F$, while $\boldsymbol{v}_{F}$ is arbitrary in $\mathbb{P}_{k}(F)^{d}$. Then, equation (43a) follows.

(ii) Mass conservation. Equation (44b) is readily obtained by replacing the definition (13b) of $D_{T}^{k}$ into the expression (15) of $b_{T}$, while $(43 \mathrm{~b})$ is obvious since $\boldsymbol{u}_{F}$ is single-valued at $F$. 
Acknowledgements. The work of D. A. Di Pietro and A. Ern was partially supported by ANR project HHOMM (ANR-15-CE40-0005). The work of F. Schieweck was partially supported by Labex Bézout.

\section{References}

[1] J. Aghili, S. Boyaval, and D. A. Di Pietro. Hybridization of mixed high-order methods on general meshes and application to the Stokes equations. Comput. Meth. Appl. Math., 15(2):111-134, 2015.

[2] C. Amrouche and V. Girault. On the existence and regularity of the solution of Stokes problem in arbitrary dimension. Proc. Japan. Acad., 67:171-175, 1991.

[3] D. Boffi, F. Brezzi, and M. Fortin. Mixed finite element methods and applications, volume 44 of Springer Series in Computational Mathematics. Springer, Berlin Heidelberg, 2013.

[4] J. Bonelle and A. Ern. Analysis of compatible discrete operator schemes for Stokes problems on polyhedral meshes. IMA J. Numer. Anal., 34(4):553-581, 2014.

[5] M. A. Case, V. J. Ervin, A. Linke, and L. G. Rebholz. A connection between Scott-Vogelius and grad-div stabilized Taylor-Hood FE approximations of the Navier-Stokes equations. SIAM J. Numer. Anal., 49(4):1461-1481, 2011.

[6] L. Cattabriga. Su un problema al contorno relativo al sistema di equazioni di Stokes. Rend. Sem. Mat. Univ. Padova, 31:308-340, 1961.

[7] P. G. Ciarlet. The finite element method for elliptic problems, volume 40 of Classics in Applied Mathematics. Society for Industrial and Applied Mathematics (SIAM), Philadelphia, PA, 2002. Reprint of the 1978 original [North-Holland, Amsterdam; MR0520174 (58 \#25001)].

[8] P. G. Ciarlet and J.-L. Lions, editors. Handbook of numerical analysis. Vol. II. Handbook of Numerical Analysis, II. North-Holland, Amsterdam, 1991. Finite element methods. Part 1, Roberts, J.E. and Thomas, J.-M.: Mixed and Hybrid Methods.

[9] B. Cockburn, D. A. Di Pietro, and A. Ern. Bridging the Hybrid High-Order and Hybridizable Discontinuous Galerkin methods. ESAIM: Math. Model Numer. Anal. (M2AN), 2015. Published online. DOI 10.1051/m2an/2015051.

[10] B. Cockburn and J. Gopalakrishnan. The derivation of hybridizable discontinuous Galerkin methods for Stokes flow. SIAM J. Numer. Anal., 47(2):1092-1125, 2009.

[11] B. Cockburn and K. Shi. Devising HDG methods for Stokes flow: An overview. Comput. E3 Fluids, 98:221-229, 2014.

[12] D. A. Di Pietro and J. Droniou. A Hybrid High-Order method for Leray-Lions elliptic equations on general meshes. Submitted. Preprint 1508.01918, August 2015.

[13] D. A. Di Pietro, J. Droniou, and A. Ern. A discontinuous-skeletal method for advection-diffusion-reaction on general meshes. SIAM J. Numer. Anal., 53(5):2135-2157, 2015.

[14] D. A. Di Pietro and A. Ern. Mathematical Aspects of Discontinuous Galerkin Methods, volume 69 of Mathématiques Es Applications. Springer, Berlin Heidelberg, 2012.

[15] D. A. Di Pietro and A. Ern. Equilibrated tractions for the Hybrid High-Order method. C. R. Acad. Sci. Paris, Ser. I, 353:279-282, 2015.

[16] D. A. Di Pietro and A. Ern. A hybrid high-order locking-free method for linear elasticity on general meshes. Comput. Meth. Appl. Mech. Engrg., 283:1-21, 2015.

[17] D. A. Di Pietro, A. Ern, and S. Lemaire. An arbitrary-order and compact-stencil discretization of diffusion on general meshes based on local reconstruction operators. Comput. Methods Appl. Math., 14(4):461-472, 2014.

[18] H. Egger and C. Waluga. $h p$ analysis of a hybrid DG method for Stokes flow. IMA J. Numer. Anal., 33(2):687-721, 2013.

[19] A. Ern and J.-L. Guermond. Theory and practice of finite elements, volume 159 of Applied Mathematical Sciences. Springer-Verlag, New York, 2004.

[20] K. J. Galvin, A. Linke, L. G. Rebholz, and N. E. Wilson. Stabilizing poor mass conservation in incompressible flow problems with large irrotational forcing and application to thermal convection. Comput. Methods Appl. Mech. Engrg., 237/240:166-176, 2012.

[21] V. Girault and P.-A. Raviart. Finite element methods for Navier-Stokes equations, volume 5 of Springer Series in Computational Mathematics. Springer-Verlag, Berlin, 1986. Theory and algorithms.

[22] E. W. Jenkins, J. Volker, A. Linke, and L. G. Rebholz. On the parameter choice in grad-div stabilization for the Stokes equations. Adv. Comput. Math., 40(2):491-516, 2014.

[23] Y. Jeon and E.-J. Park. New locally conservative finite element methods on a rectangular mesh. Numer. Math., 123(1):97-119, 2013.

[24] Y. Jeon, E.-J. Park, and D. Sheen. A hybridized finite element method for the Stokes problem. Computers and Mathematics with Applications, 68:2222-2232, 2014.

[25] V. John, A. Linke, C. Merdon, M. Neilan, and L. Rebholz. On the divergence constraint in mixed finite element methods for incompressible flows. Submitted. WIAS Preprint 2177, 2015.

[26] R.J. Labeur and G.N. Wells. Energy stable and momentum conserving hybrid fininte element method for the incompressible Navier-Stokes equations. SIAM J. Sci. Comput., 34(2):A889-A913, 2012. 
[27] C. Lehrenfeld and J. Schöberl. High order exactly divergence-free hybrid discontinuous Galerkin methods for unsteady incompressible flows. Submitted. ASC Report No. 27/2015, TU Vienna, 2015.

[28] A. Linke. Collision in a cross-shaped domain - a steady 2d Navier-Stokes example demonstrating the importance of mass conservation in CFD. Comput. Methods Appl. Mech. Engrg., 198(41-44):3278-3286, 2009.

[29] A. Linke. A divergence-free velocity reconstruction for incompressible flows. C. R. Math. Acad. Sci. Paris, 350(1718):837-840, 2012 .

[30] A. Linke. On the role of the Helmholtz decomposition in mixed methods for incompressible flows and a new variational crime. Comput. Methods Appl. Mech. Engrg., 268:782-800, 2014.

[31] A. Linke, G. Matthies, and L. Tobiska. Robust arbitrary order mixed finite element methods for the incompressible stokes equations with pressure independent velocity errors. M2AN, 50(1):289-309, 2016.

[32] A. Linke and C. Merdon. On velocity errors due to irrotational forces in the Navier-Stokes momentum balance. Journal of Computational Physics, 313:654-661, 2016.

[33] K. Lipnikov and G. Manzini. A high-order mimetic method on unstructured polyhedral meshes for the diffusion equation. J. Comput. Phys., 272:360-385, 2014.

[34] L. Mu, X. Wang, and X. Ye. A modified weak Galerkin finite element method for the Stokes equations. J. Comput. Phys., 275:79-90, 2015.

[35] N.C. Nguyen, J. Peraire, and B. Cockburn. A hybridizable discontinuous Galerkin methods for Stokes flow. Comput. Methods Appl. Mech. Engrg., 199:582-597, 2010.

[36] M. Olshanskii, G. Lube, T. Heister, and J. Löwe. Grad-div stabilization and subgrid pressure models for the incompressible Navier-Stokes equations. Comput. Methods Appl. Mech. Engrg., 198(49-52):3975-3988, 2009.

[37] M. A. Olshanskii and A. Reusken. Grad-div stabilization for Stokes equations. Math. Comp., 73(248):1699-1718, 2004.

[38] J. R. Shewchuk. Triangle: Engineering a 2D Quality Mesh Generator and Delaunay Triangulator. In Ming C. Lin and Dinesh Manocha, editors, Applied Computational Geometry: Towards Geometric Engineering, volume 1148 of Lecture Notes in Computer Science, pages 203-222. Springer-Verlag, May 1996. From the First ACM Workshop on Applied Computational Geometry.

[39] H. Si. Tetgen, a delaunay-based quality tetrahedral mesh generator. ACM Trans. Math. Softw., 41(2):11:1-11:36, 2015.

[40] T. Streckenbach, J. Fuhrmann, H. Langmach, and M. Uhle. Pdelib - a software toolbox for numerical computations. http://www.wias-berlin.de/software/pdelib/, 2009.

[41] B. Wang and B.C. Khoo. Hybridizable discontinuous Galerkin method (HDG) for Stokes interface flow. J. Comput. Phys., 247:262-278, 2013. 


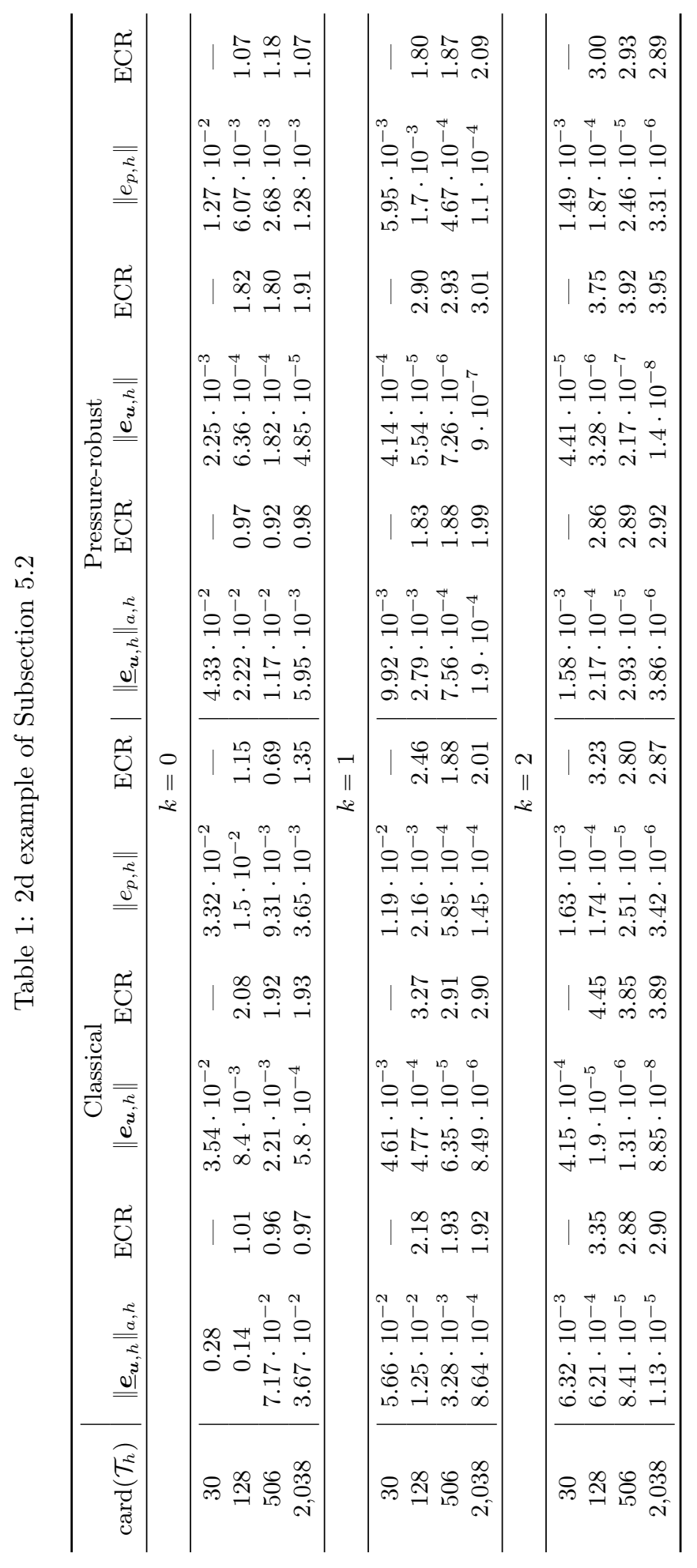




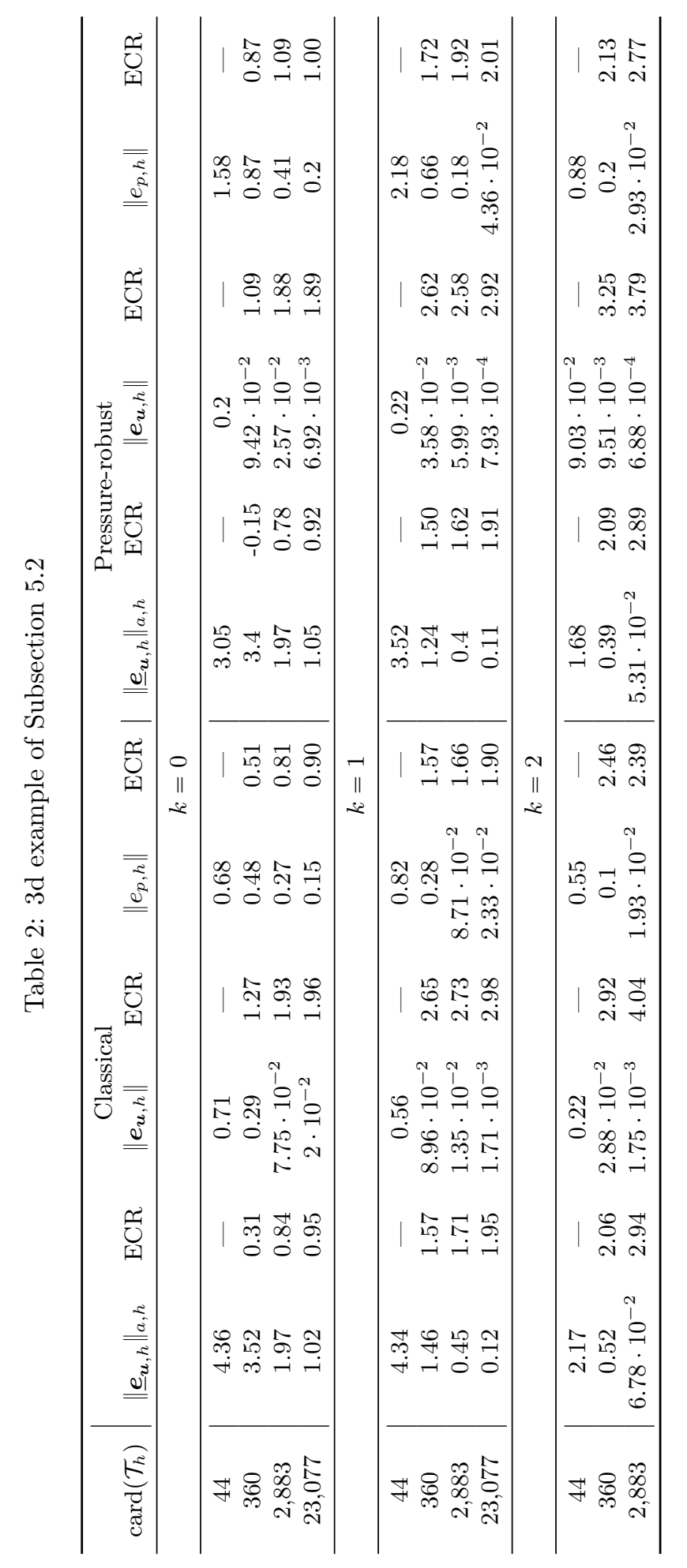

